\section{OPEN ACCESS}

Edited by:

Qiaobing Huang,

Southern Medical University, China

Reviewed by:

Lin Yao,

Guangzhou University of Chinese

Medicine, China

You Yun,

China Academy of Chinese Medical

Sciences, China

*Correspondence:

Guo-qing Zheng

gq_zheng@sohu.com

Yan Wang

wywzchina@sina.com

tThese authors have contributed equally to this work

Specialty section:

This article was submitted to

Vascular Physiology,

a section of the journal

Frontiers in Physiology

Received: 25 July 2019 Accepted: 25 September 2019

Published: 16 October 2019

Citation:

Zhu P-C, Tong Q, Zhuang Z, Wang $Z-H$, Deng $L-H$, Zheng $G$ and

Wang Y (2019) Ginkgolide B for

Myocardial Ischemia/Reperfusion Injury: A Preclinical Systematic Review and Meta-Analysis.

Front. Physiol. 10:1292. doi: 10.3389/fphys.2019.01292

\title{
Ginkgolide B for Myocardial Ischemia/Reperfusion Injury: A Preclinical Systematic Review and Meta-Analysis
}

\author{
Peng-Chong Zhu ${ }^{1+}$, Qiang Tong ${ }^{1+}$, Zhuang Zhuang ${ }^{1}$, Zi-Hao Wang ${ }^{1}$, Li-Hui Deng ${ }^{1}$, \\ Guo-qing Zheng ${ }^{2 *}$ and Yan Wang ${ }^{1 *}$
}

${ }^{1}$ Department of Cardiology, The Second Affiliated Hospital and Yuying Children's Hospital of Wenzhou Medical University, Wenzhou, China, ${ }^{2}$ Department of Neurology, The Second Affiliated Hospital and Yuying Children's Hospital of Wenzhou Medical University, Wenzhou, China

Ginkgolide B (GB) is an extract of dried Ginkgo biloba leaves and possesses various pharmacological activities in the cardiovascular system. Herein, we aim to assess the available preclinical evidence and possible mechanisms of GB for myocardial ischemia/reperfusion injury. The study quality score was assessed using the CAMARADES 10-item checklist. Rev-Man 5.3 software was used for data analyses. Nineteen studies with total 437 animals were included for analysis. Meta-analyses indicated that GB interventions significantly reduce myocardial infarct size and cardiac markers when compared with control $(P<0.05)$. The possible mechanisms via which GB exerts cardioprotective effects are mainly associated with anti-oxidation, anti-inflammation, anti-apoptosis, and improvement of energy metabolism. Our study indicates that GB might be a promising cardioprotective agent for myocardial ischemia/reperfusion injury and may contribute to future clinical trial design.

Keywords: Ginkgolide B, myocardial ischemia/reperfusion injury, possible mechanisms, preclinical evidence, meta-analysis

\section{INTRODUCTION}

Coronary heart disease (CHD) is the leading cause of death and disability globally (Ibanez et al., 2015). Acute myocardial infarction (AMI) is one critical clinical presentation of coronary artery disease (Yellon and Hausenloy, 2007). Early restoration of blood flow through thrombolysis or primary percutaneous coronary intervention has been proved to be the most effective method to rescue ischemic myocardium, reduce infarct size, and improve the clinical outcomes (Frank et al., 2012). However, the process of reperfusion paradoxically lead to myocardial ischemia/reperfusion (I/R) injury such as myocardial necrosis, stunning, heart failure, no-reflow and reperfusion arrhythmias (Binder et al., 2015), which would undermine the benefits of myocardial reperfusion (Minamino, 2012). Myocardial I/R injury is an intricate phenomenon involved in many factors, all conducing to the final damage inflicted on the cardiomyocyte (Bulluck et al., 2016). Over the past 3 decades, numerous therapeutic strategies, both pharmacologic and non-pharmacologic, have been proposed to ameliorate the myocardial I/R injury in animal experiments. However, the basic research yielded inconclusive results or failed to translate into the clinical setting efficiently (Eltzschig and Eckle, 2011). Hence, it is worthy to find a novel cardioprotective therapeutic intervention to prevent further tissue injury caused by reperfusion. 


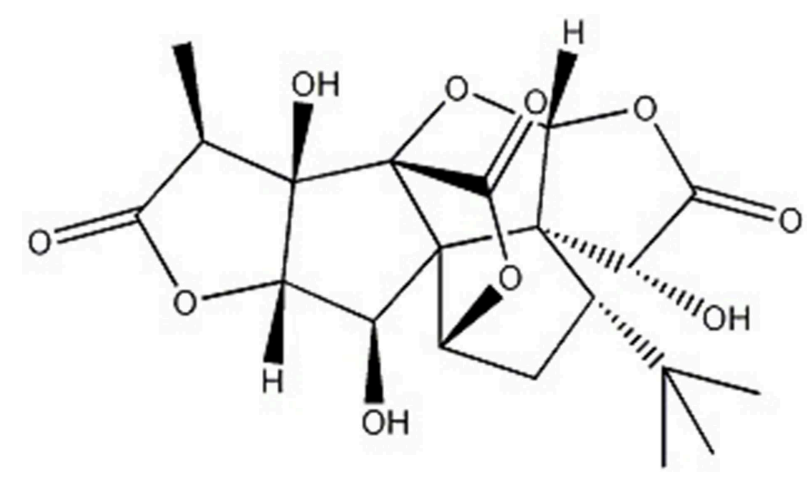

FIGURE 1 | Chemical structures of $\mathrm{GB}\left(\mathrm{C}_{20} \mathrm{H}_{24} \mathrm{O}_{10}\right)$.

Ginkgo biloba L., known as "living fossil" in plant kingdom (Jacobs and Browner, 2000), its leaves were applied as a traditional Chinese medicine (TCM) for the management of cardiovascular disorders for hundreds of years because of its properties of promoting blood circulation to remove blood stasis (Zhang et al., 2016) and activating meridians to stop pain ( $\mathrm{Li}$ et al., 2017). Ginkgolide B (GB) (Figure 1), an extract of dried Ginkgo biloba leaves, was discovered as a natural antagonist of platelet activating factor (PAF) with various biological and pharmacological functions, including anti-allergic (Bilia, 2002), anti-inflammation (Xia and Fang, 2007), and anti-oxidation (Maclennan et al., 2002). Thus, it was widely used for I/R diseases including AMI.

The animal research has its special primary purpose in improving the understanding of physiologic and pathologic processes (Briel et al., 2013). Systematic reviews of preclinical studies play an important role in healthcare practice which can help promote the translation of findings from animal studies to clinical trials rapidly and appropriately, and avoid unintended waste of time (Sandercock and Roberts, 2002). In addition, preclinical systematic review can contribute to illustrate mechanisms, synthesize the available evidence, support clinical decision-making, and improve the methodological quality of experiments and guide health policy (Roberts et al., 2002). Many animal studies have hitherto investigated the role of GB in myocardial I/R injury. However, the efficacy and mechanisms of GB for myocardial I/R injury have not been systematically appraised and summarized. In the present study, we performed a systematic review for these animal studies to assess the available preclinical evidence and possible mechanisms of GB in treating myocardial I/R injury.

\section{METHODS}

\section{Data Sources and Search Strategy}

The PRISMA checklist is available as supporting information (Stewart et al., 2015). We searched for animal studies of GB for myocardial $\mathrm{I} / \mathrm{R}$ injury in Chinese National Knowledge Infrastructure (CNKI), EMBASE, PubMed, VIP information database, Web of Science, and Wanfang data Information
Site published up to the end of April 2019. The following search phrases were used: "Yinxingneizhi B (MeSH Terms) OR Ginkgolide B (Title/Abstract)" AND "Myocardial infarction OR Myocardial ischemia OR Myocardial I/R". The references of the included articles cited were searched manually for the eligible studies.

\section{Eligibility Criteria}

Studies were included for analysis when they meet: (1) animal studies assessing the administration of GB for myocardial I/R model in vivo or ex vivo except cell studies, case reports, reviews, abstracts, and comments; (2) analyzed intervention received GB treatment merely; comparator intervention received vehicle or no treatment; (3) primary outcomes were myocardial infarction (MI) size, myocardial injury marker, ST-segment changes in electrocardiograms, or cardiac function indicators in cardiac ultrasound, while secondary outcomes were serum indices or protein levels relative to the mechanisms of GB in myocardial I/R injury. The studies compared with other TCM, administered other additional pharmacological treatment, without predetermined outcome index and without involving animal models of myocardial I/R injury were excluded.

\section{Data Extraction}

The following details were recorded: (1) first author's name and year of publication; (2) animal information, including species, gender, number, and weight; (3) methods for myocardial I/R model establishment, including I/R duration and coronary artery occluded; (4) method for anaesthetic induction; (5) the information of treatment, including time of intervention, dose, and route of administration; (6) the primary outcome measures, secondary outcome measures and its intergroup differences. Only the data of final test was collected when outcome measures were tested at different time points. The result of highest dose was extracted if various doses of drugs were used in the study. If some data were only expressed as graphs, we reached out to the authors for raw data. Measure numerical values by digital ruler software when no response was got from authors.

\section{Risk of Bias in Individual Studies}

Two authors independently evaluated the quality of study using the 10-point scoring scale (Macleod et al., 2004): A, peerreviewed publication; $\mathrm{B}$, statement of temperature control; $\mathrm{C}$, random allocation to groups; $\mathrm{D}$, allocation concealment; $\mathrm{E}$, blinded assessment of outcome; F, use of anesthetic without significant intrinsic cardioprotective activity; G, appropriate animal model (aged, diabetic, or hypertensive); H, sample size calculation; I, compliance with animal welfare regulations; and J, statement of potential conflict of interests. Study selection, data extraction, and quality evaluation of studies were independently performed by two authors, and the disputes were discussed in group discussion.

\section{Statistical Analysis}

All outcome measures were treated as continuous data. Every outcome with at least 2 available studies was analyzed with RevMan V.5.3 software. Outcomes were presented as 
Standardized mean difference (SMD) with 95\% confidence interval (CI). Heterogeneity among the studies was evaluating using the $I$-square $\left(I^{2}\right)$ statistics test. If $I^{2}>50 \%$, a random effects model was applied; otherwise, a fixed effects model was applied. $P$-value $<0.05$ was considered significant. A forest plot was created to summarize the meta-analysis study results.

\section{RESULTS}

The initial search yielded a total of 213 studies. After excluding 137 irrelevant and duplicated studies, the full texts of 76 studies were screened. In the process, 57 studies were excluded according to the inclusion and exclusion criteria. Ultimately, 19 studies (Liebgott et al., 2000; Jiao et al., 2002; Rioufol et al., 2003; Billottet et al., 2005; Zhang et al., 2005, 2018; Hao et al., 2009, 2013, 2016; Zhao et al., 2014; Pei et al., 2015; Xiong and Wei, 2015; Bai et al., 2016; Cao et al., 2016; Hao and Dong, 2017; Meng, 2017; Zhuang et al., 2017; Chai et al., 2018; Yang et al., 2018) were selected. A detailed step of the selection process is shown in Figure 2.

\section{Characteristics of Included Studies}

Twelve Chinese studies (Zhang et al., 2005; Hao et al., 2013, 2016; Zhao et al., 2014; Xiong and Wei, 2015; Bai et al., 2016; Cao et al., 2016; Hao and Dong, 2017; Meng, 2017; Zhuang et al., 2017; Chai et al., 2018; Yang et al., 2018) and 7 English studies (Liebgott et al., 2000; Jiao et al., 2002; Rioufol et al., 2003; Billottet et al., 2005; Hao et al., 2009; Pei et al., 2015; Zhang et al., 2018) were published between 1996 and 2018. Male Sprague-Dawley rats (11 studies) (Hao et al., 2009, 2013, 2016; Xiong and Wei, 2015; Bai et al., 2016; Hao and Dong, 2017; Meng, 2017; Zhuang et al., 2017; Chai et al., 2018; Yang et al., 2018; Zhang et al., 2018), male/female Sprague-Dawley rats (2 studies) (Zhao et al., 2014; Cao et al., 2016), male Wistar rats (4 studies) (Liebgott et al., 2000; Jiao et al., 2002; Billottet et al., 2005; Pei et al., 2015), male/female Wistar rats (1 study) (Zhang et al., 2005), and male/female Farm pigs (1 study) (Rioufol et al., 2003)were utilized. Chloral hydrate was used in 4 studies (Zhao et al., 2014; Xiong and Wei, 2015; Yang et al., 2018; Zhang et al., 2018); pentobarbital sodium in 7 studies (Liebgott et al., 2000; Billottet et al., 2005; Hao et al., 2013, 2016; Pei et al., 2015; Cao et al., 2016; Hao and Dong, 2017); urethane in 6 studies (Jiao et al., 2002; Zhang et al., 2005; Bai et al., 2016; Meng, 2017; Zhuang et al., 2017; Chai et al., 2018); pentobarbitalin 2 studies (Rioufol et al., 2003; Hao et al., 2009) for anesthesia. Ligating the coronary artery was to induce the model of $\mathrm{I} / \mathrm{R}$, of which the left anterior descending coronary artery (LAD) was in 11 studies (Jiao et al., 2002; Rioufol et al., 2003; Pei et al., 2015; Xiong and Wei, 2015; Bai et al., 2016; Cao et al., 2016; Meng, 2017; Zhuang et al., 2017; Chai et al., 2018; Yang et al., 2018; Zhang et al., 2018). Others were established by the langendorff method (Liebgott et al., 2000; Billottet et al., 2005; Zhang et al., 2005; Hao et al., 2009, 2013, 2016; Zhao et al., 2014; Hao and Dong, 2017). MI size as outcome was reported in 9 studies (Jiao et al., 2002; Hao et al., 2009, 2016; Xiong and Wei, 2015; Bai et al., 2016; Cao et al., 2016; Meng, 2017; Zhuang

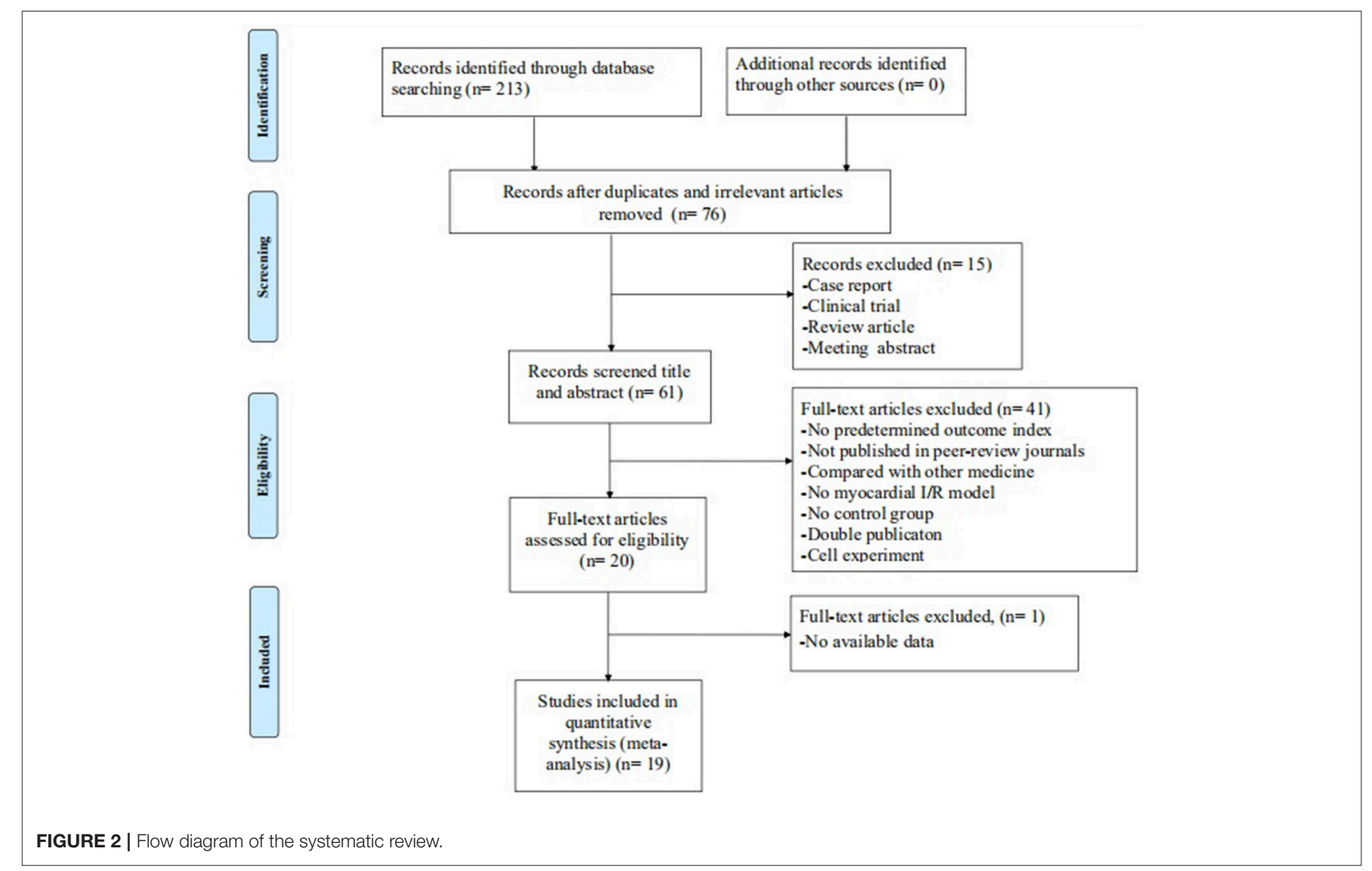




\begin{tabular}{|c|c|c|c|c|c|c|c|c|}
\hline Study (years) & $\begin{array}{l}\text { Species (Sex, } n \\
=\text { experimental/ } \\
\text { control group) }\end{array}$ & Weight & Model (method) & Anesthetic & $\begin{array}{l}\text { Treatment group } \\
\text { (method to GB) }\end{array}$ & Control group & Outcome index (time) & $\begin{array}{l}\text { Intergroup } \\
\text { differences }\end{array}$ \\
\hline $\begin{array}{l}\text { Zhuang et al., } \\
2017 \text { (1) }\end{array}$ & $\begin{array}{l}\text { SD rats } \\
\text { (male, 20/20) }\end{array}$ & $220-260 \mathrm{~g}$ & $\begin{array}{l}\text { Block LAD for } \\
30 \text { min then } \\
\text { reperfusion }\end{array}$ & Urethane (650 mg/kg) & $\begin{array}{l}\text { Intraperitoneal injection GB ( } 60 \\
\left.\mathrm{mg} / \mathrm{kg}^{\star} \mathrm{d}\right) \text {, once a day, for } 7 \text { days, } \\
\text { after establishing model }\end{array}$ & $\begin{array}{l}\text { Intraperitoneal injection isasteric } \\
\text { normal saline, once a day, for } 7 \\
\text { days, after establishing model }\end{array}$ & $\begin{array}{l}\text { 1. Infarct size (AAI/LVA) } \\
\text { 2. Apoptosis index } \\
\text { 3. Bcl-2 } \\
\text { 4. Bax } \\
\text { 5. Caspase-3 }\end{array}$ & $\begin{array}{l}\text { 1. } P<0.01 \\
\text { 2. } P<0.01 \\
\text { 3. } P<0.01 \\
\text { 4. } P<0.01 \\
\text { 5. } P<0.01\end{array}$ \\
\hline $\begin{array}{l}\text { Xiong and Wei, } \\
2015 \text { (2) }\end{array}$ & $\begin{array}{l}\text { SD rats } \\
\text { (male, 20/20) }\end{array}$ & $250-270 \mathrm{~g}$ & $\begin{array}{l}\text { Block of LAD for } \\
30 \text { min then } \\
\text { reperfusion } \\
\text { for } 48 \mathrm{~h}\end{array}$ & $7 \%$ chloral hydrate & $\begin{array}{l}\text { Gavaged with GB }\left(60 \mathrm{mg} / \mathrm{kg}^{*} \mathrm{~d}\right) \text {, } \\
\text { once a day, for } 7 \text { days, before } \\
\text { establishing model }\end{array}$ & $\begin{array}{l}\text { Gavaged with isasteric normal } \\
\text { saline, once a day, for } 7 \text { days, } \\
\text { before establishing model }\end{array}$ & $\begin{array}{l}\text { 1. Infarct size (AAI/LVA) } \\
\text { 2. EF } \\
\text { 3. FS } \\
\text { 4. Bcl-2 } \\
\text { 5. Bax } \\
\text { 6. p-ErK } \\
\text { 7.p-JNK } \\
\text { 8. p-p38MAPK }\end{array}$ & $\begin{array}{l}\text { 1. } P<0.01 \\
\text { 2. } P<0.01 \\
\text { 3. } P<0.01 \\
\text { 4. } P<0.01 \\
\text { 5. } P<0.01 \\
\text { 6. } P<0.01 \\
\text { 7. } P<0.01 \\
\text { 8. } P<0.01\end{array}$ \\
\hline Cao et al., 2016 (3) & $\begin{array}{l}\text { SD rats } \\
\text { (male/female, } \\
\text { 10/10) }\end{array}$ & $200-240 \mathrm{~g}$ & $\begin{array}{l}\text { Block LAD for } \\
30 \text { min then } \\
\text { reperfusion for } 2 \mathrm{~h}\end{array}$ & $\begin{array}{l}\text { Pentobarbital sodium } \\
\text { ( } 45 \mathrm{mg} / \mathrm{kg} \text { ) }\end{array}$ & $\begin{array}{l}\text { Tail intravenous injection GB ( } 8 \\
\mathrm{mg} / \mathrm{kg}), 1 \mathrm{~h} \text { before establishing } \\
\text { model }\end{array}$ & $\begin{array}{l}\text { Tail intravenous injection isasteric } \\
\text { normal saline, } 1 \mathrm{~h} \text { before } \\
\text { establishing model }\end{array}$ & $\begin{array}{l}\text { 1. Infarct size (AAI/LVA) } \\
\text { 2. CK } \\
\text { 3. LDH } \\
\text { 4. SOD } \\
\text { 5. MDA }\end{array}$ & $\begin{array}{l}\text { 1. } P<0.05 \\
\text { 2. } P<0.01 \\
\text { 3. } P<0.01 \\
\text { 4. } P<0.01 \\
\text { 5. } P<0.01\end{array}$ \\
\hline Meng, 2017 (4) & $\begin{array}{l}\text { SD rats } \\
\text { (male, 20/20) }\end{array}$ & $220-260 \mathrm{~g}$ & $\begin{array}{l}\text { Block LAD for } \\
30 \text { min then } \\
\text { reperfusion }\end{array}$ & Urethane (650 mg/kg) & $\begin{array}{l}\text { Intraperitoneal injection GB ( } 60 \\
\left.\mathrm{mg} / \mathrm{kg}^{*} \mathrm{~d}\right) \text { ), once a day, for } 7 \text { days, } \\
\text { after establishing model }\end{array}$ & $\begin{array}{l}\text { Intraperitoneal injection isasteric } \\
\text { normal saline, once a day, for } 7 \\
\text { days, after establishing model }\end{array}$ & $\begin{array}{l}\text { 1. Infarct size (AAI/LVA) } \\
\text { 2. EF } \\
\text { 3. FS } \\
\text { 4. LVIDd } \\
\text { 5. LVIDs } \\
\text { 6. SV } \\
\text { 7. Apoptosis index } \\
\text { 8. AST } \\
\text { 9. CK } \\
\text { 10. LDH }\end{array}$ & $\begin{array}{l}\text { 1. } P<0.01 \\
\text { 2. } P<0.01 \\
\text { 3. } P<0.01 \\
\text { 4. } P<0.01 \\
\text { 5. } P<0.01 \\
\text { 6. } P<0.01 \\
\text { 7. } P<0.01 \\
\text { 8. } P<0.01 \\
\text { 9. } P<0.01\end{array}$ \\
\hline Bai et al., 2016 (5) & $\begin{array}{l}\text { SD rats } \\
\text { (male, 20/20) }\end{array}$ & $220-260 \mathrm{~g}$ & $\begin{array}{l}\text { Block LAD for } \\
30 \text { min then } \\
\text { reperfusion }\end{array}$ & Urethane (650 mg/kg) & $\begin{array}{l}\text { Intraperitoneal injection GB ( } 60 \\
\text { mg/d), once a day, for } 7 \text { days, after } \\
\text { establishing model }\end{array}$ & $\begin{array}{l}\text { Intraperitoneal injection isasteric } \\
\text { normal saline, once a day, for } 7 \\
\text { days, after establishing model }\end{array}$ & $\begin{array}{l}\text { 1. Infarct size (AAI/LVA) } \\
\text { 2. Apoptosis index } \\
\text { 3. SOD } \\
\text { 4. GSH-Px } \\
\text { 5. CAT } \\
\text { 6. MDA } \\
\text { 7. AST } \\
\text { 8. CK } \\
\text { 9. LDH } \\
\text { 10. NF-kB }\end{array}$ & $\begin{array}{l}\text { 1. } P<0.01 \\
\text { 2. } P<0.01 \\
\text { 3. } P<0.01 \\
\text { 4. } P<0.01 \\
\text { 5. } P<0.01 \\
\text { 6. } P<0.01 \\
\text { 7. } P<0.01 \\
\text { 8. } P<0.01 \\
\text { 9. } P<0.01 \\
\text { 10. } P<0.01\end{array}$ \\
\hline $\begin{array}{l}\text { Chai et al., } 2018 \\
\text { (6) }\end{array}$ & $\begin{array}{l}\text { SD rats } \\
\text { (male, 20/20) }\end{array}$ & $220-260 \mathrm{~g}$ & $\begin{array}{l}\text { Block LAD for } \\
30 \text { min then } \\
\text { reperfusion for } 1 \mathrm{~h}\end{array}$ & Urethane (1 g/kg) & $\begin{array}{l}\text { Intraperitoneal injection GB (60 } \\
\mathrm{mg} / \mathrm{kg}^{*} \mathrm{~d} \text { ), once a day, for } 7 \text { days, } \\
\text { before establishing model }\end{array}$ & $\begin{array}{l}\text { Intraperitoneal injection ( } 18 \\
\mathrm{mg} / \mathrm{kg}^{\star} \mathrm{d} \text { ) normal saline, once a } \\
\text { day, for } 7 \text { days, before } \\
\text { establishing model }\end{array}$ & $\begin{array}{l}\text { 1. Arrhythmia score } \\
\text { 2. SOD } \\
\text { 3. GSH-Px } \\
\text { 4. MDA }\end{array}$ & $\begin{array}{l}\text { 1. } P<0.01 \\
\text { 2. } P<0.01 \\
\text { 3. } P<0.01 \\
\text { 4. } P<0.01\end{array}$ \\
\hline $\begin{array}{l}\text { Yang et al., } 2018 \\
\text { (7) }\end{array}$ & $\begin{array}{l}\text { SD rats } \\
\text { (male, } 7 / 7)\end{array}$ & $180-220 \mathrm{~g}$ & $\begin{array}{l}\text { Block LAD for } \\
30 \text { min then } \\
\text { reperfusion for } 2 \mathrm{~h}\end{array}$ & $10 \%$ chloral hydrate & $\begin{array}{l}\text { Intraperitoneal injected with GB (60 } \\
\text { mg/kg), } 30 \text { min before establishing } \\
\text { model }\end{array}$ & $\begin{array}{l}\text { Intraperitoneal injection isasteric } \\
\text { normal saline, } 30 \mathrm{~min} \text { before } \\
\text { establishing model }\end{array}$ & $\begin{array}{l}\text { 1. LVSP } \\
\text { 2. LVDP } \\
\text { 3. }+\mathrm{dP} / \mathrm{dtmax} \\
\text { 4. }-\mathrm{dP} / \mathrm{dtmax} \\
\text { 5. Caspase-3 }\end{array}$ & $\begin{array}{l}\text { 1. } P<0.05 \\
\text { 2. } P<0.05 \\
\text { 3. } P<0.05 \\
\text { 4. } P<0.05 \\
\text { 5. } P<0.05\end{array}$ \\
\hline
\end{tabular}


TABLE 1 | Continued

\begin{tabular}{|c|c|c|c|c|c|c|c|c|}
\hline Study (years) & $\begin{array}{l}\text { Species (Sex, } n \\
=\text { experimental/ } \\
\text { control group) }\end{array}$ & Weight & Model (method) & Anesthetic & $\begin{array}{l}\text { Treatment group } \\
\text { (method to GB) }\end{array}$ & Control group & Outcome index (time) & $\begin{array}{l}\text { Intergroup } \\
\text { differences }\end{array}$ \\
\hline $\begin{array}{l}\text { Jiao et al., } 2002 \\
\text { (8) }\end{array}$ & $\begin{array}{l}\text { Wistar rats } \\
\text { (male, 12/12) }\end{array}$ & $250-300 \mathrm{~g}$ & $\begin{array}{l}\text { Block LAD for } \\
30 \text { min then } \\
\text { reperfusion for } 3 \mathrm{~h}\end{array}$ & Urethane $(1 \mathrm{~g} / \mathrm{kg})$ & $\begin{array}{l}\text { Intraperitoneal injection GB ( } 5 \\
\mathrm{mg} / \mathrm{kg} \text { ), } 25 \text { and } 10 \text { min before } \\
\text { establishing model respectively }\end{array}$ & $\begin{array}{l}\text { Intraperitoneal injection isasteric } \\
\text { normal saline, } 25 \text { and } 10 \mathrm{~min} \\
\text { before establishing model } \\
\text { respectively }\end{array}$ & $\begin{array}{l}\text { 1. Infarct size (AAl/AAR) } \\
\text { 2. LVSP } \\
\text { 3. }+\mathrm{dP} / \mathrm{dtmax} \\
\text { 4. }-\mathrm{dP} / \mathrm{dtmax} \\
\text { 5. Apoptosis index }\end{array}$ & $\begin{array}{l}\text { 1. } P<0.05 \\
\text { 2. } P>0.05 \\
\text { 3. } P<0.05 \\
\text { 4. } P>0.05 \\
\text { 5. } P<0.05\end{array}$ \\
\hline $\begin{array}{l}\text { Rioufol et al., } 2003 \\
\text { (9) }\end{array}$ & $\begin{array}{l}\text { Farm pigs } \\
\text { (male/female, 6/7) }\end{array}$ & $27-28 \mathrm{Kg}$ & $\begin{array}{l}\text { Block LAD for } \\
10 \text { min then } \\
\text { reperfusion for } 3 \mathrm{~h}\end{array}$ & $\begin{array}{l}\text { Pentobarbital (15 } \\
\mathrm{mg} / \mathrm{kg})\end{array}$ & $\begin{array}{l}\text { Intravenous injection GB ( } 0.3 \\
\mathrm{mg} / \mathrm{kg}), 48 \mathrm{~h} \text { and } 24 \mathrm{~h} \text { before } \\
\text { establishing model }\end{array}$ & $\begin{array}{l}\text { Intravenous injection isasteric } \\
\text { normal saline, } 48 \mathrm{~h} \text { and } 24 \mathrm{~h} \\
\text { before establishing model }\end{array}$ & $\begin{array}{l}\text { 1. HR } \\
\text { 2. Mean arterial pressure } \\
\text { 3. Segment shortening }\end{array}$ & $\begin{array}{l}\text { 1. } P>0.05 \\
\text { 2. } P>0.05 \\
\text { 3. } P>0.05\end{array}$ \\
\hline $\begin{array}{l}\text { Pei et al., } 2015 \\
\text { (10) }\end{array}$ & $\begin{array}{l}\text { Wistar rats } \\
\text { (male, 10/12) }\end{array}$ & $280-400 \mathrm{~g}$ & $\begin{array}{l}\text { Block LAD for } \\
5 \text { min then } \\
\text { reperfusion for } \\
10 \text { min }\end{array}$ & $\begin{array}{l}\text { pentobarbitalsodium } \\
(4.5 \mathrm{mg} / \mathrm{kg})\end{array}$ & $\begin{array}{l}\text { Intravenous injection GB ( } 15 \\
\mathrm{mg} / \mathrm{kg} \text { ), } 10 \text { min before establishing } \\
\text { model }\end{array}$ & $\begin{array}{l}\text { Intravenous injection isasteric } \\
\text { normal saline, } 10 \mathrm{~min} \text { before } \\
\text { establishing model }\end{array}$ & $\begin{array}{l}\text { 1. LVSP } \\
\text { 2. Arrhythmia score } \\
\text { 3. }+\mathrm{dP} / \mathrm{dtmax} \\
\text { 4. }-\mathrm{dP} / \mathrm{dtmax} \\
\text { 5. LDH }\end{array}$ & $\begin{array}{l}\text { 1. } P<0.01 \\
\text { 2. } P<0.01 \\
\text { 3. } P<0.01 \\
\text { 4. } P<0.01 \\
\text { 5. } P<0.05\end{array}$ \\
\hline $\begin{array}{l}\text { Hao et al., } 2009 \\
\text { (11) }\end{array}$ & $\begin{array}{l}\text { SD rats } \\
\text { (male, } 7 / 7)\end{array}$ & $220-250 \mathrm{~g}$ & $\begin{array}{l}\text { Langendorff Model } \\
30 \text { min stabilization } \\
\text { I/R (30 } \\
\mathrm{min} / 120 \mathrm{~min})\end{array}$ & $\begin{array}{l}\text { Pentobarbital (150 } \\
\mathrm{mg} / \mathrm{kg})\end{array}$ & $\begin{array}{l}\text { GB (10uM), for } 10 \mathrm{~min} \text { before } \\
\text { ischemia }\end{array}$ & No treatment & $\begin{array}{l}\text { 1. Infarct size (AAI/LVA) } \\
\text { 2. HR } \\
\text { 3. LVSP } \\
\text { 4. LVEDP } \\
\text { 5. }+\mathrm{dP} / \mathrm{dtmax} \\
\text { 6. }-\mathrm{dP} / \mathrm{dtmax} \\
\text { 7. } \mathrm{LDH} \\
\text { 8. Bcl-2 } \\
\text { 9. Bax }\end{array}$ & $\begin{array}{l}\text { 1. } P<0.05 \\
\text { 2. } P<0.01 \\
\text { 3. } P>0.05 \\
\text { 4. } P>0.05 \\
\text { 5. } P<0.05 \\
\text { 6. } P<0.05 \\
\text { 7. } P<0.05 \\
\text { 8. } P>0.05 \\
\text { 9. } P>0.05\end{array}$ \\
\hline $\begin{array}{l}\text { Hao and Dong, } \\
2017 \text { (12) }\end{array}$ & $\begin{array}{l}\text { SD rats } \\
\text { (male, 10/10) }\end{array}$ & $350-500 \mathrm{~g}$ & $\begin{array}{l}\text { Langendorff Model } \\
30 \mathrm{~min}, 1 / \mathrm{R}(30 \\
\mathrm{min} / 60 \mathrm{~min})\end{array}$ & $\begin{array}{l}1 \% \text { pentobarbital } \\
\text { sodium }\end{array}$ & $\begin{array}{l}\mathrm{GB}(10 \mu \mathrm{mol} / \mathrm{L}) \text {, first } 10 \mathrm{~min} \text { of } \\
\text { reperfusion }\end{array}$ & No treatment & $\begin{array}{l}\text { 1. } \Delta \text { LVP (LVSP -LVEDP) } \\
\text { 2. }+\mathrm{dP} / \mathrm{dtmax} \\
\text { 3. }-\mathrm{dP} / \mathrm{dtmax} \\
\text { 4. LDH }\end{array}$ & $\begin{array}{l}\text { 1. } P<0.05 \\
\text { 2. } P<0.05 \\
\text { 3. } P<0.05 \\
\text { 4. } P<0.05\end{array}$ \\
\hline $\begin{array}{l}\text { Hao et al., } 2013 \\
\text { (13) }\end{array}$ & $\begin{array}{l}\text { SD rats } \\
\text { (male, 8/8) }\end{array}$ & $200-250 \mathrm{~g}$ & $\begin{array}{l}\text { Langendorff Model } \\
30 \text { min stabilization } \\
\text { I/R (20 min/40 min) }\end{array}$ & $\begin{array}{l}1 \% \text { pentobarbital } \\
\text { sodium }\end{array}$ & $\begin{array}{l}\text { GB }(10 \mu \mathrm{mol} / \mathrm{L}) \text {, for } 10 \mathrm{~min} \text { before } \\
\text { ischemia }\end{array}$ & No treatment & $\begin{array}{l}\text { 1. } \Delta \text { LVP (LVSP -LVEDP) } \\
\text { 2. }+\mathrm{dP} / \mathrm{dtmax} \\
\text { 3. }-\mathrm{dP} / \mathrm{dtmax} \\
\text { 4. } \mathrm{HR} \\
\text { 5. LDH } \\
\text { 6. SOD }\end{array}$ & $\begin{array}{l}\text { 1. } P<0.01 \\
\text { 2. } P<0.05 \\
\text { 3. } P<0.01 \\
\text { 4. } P<0.05 \\
\text { 5. } P<0.01 \\
\text { 6. } P<0.05\end{array}$ \\
\hline $\begin{array}{l}\text { Hao et al., } 2016 \\
\text { (14) }\end{array}$ & $\begin{array}{l}\text { SD rats } \\
\text { (male, 10/10) }\end{array}$ & $350-500 \mathrm{~g}$ & $\begin{array}{l}\text { Langendorff Model } \\
30 \text { min stabilization } \\
\text { I/R (30 min/90 min) }\end{array}$ & $\begin{array}{l}1 \% \text { pentobarbital } \\
\text { sodium }\end{array}$ & $\begin{array}{l}\mathrm{GB}(10 \mu \mathrm{mol} / \mathrm{L}) \text {, first } 10 \mathrm{~min} \text { of } \\
\text { reperfusion }\end{array}$ & No treatment & $\begin{array}{l}\text { 1. Infarct size (AAI/LVA) } \\
\text { 2. LVSP } \\
\text { 3. LVEDP } \\
\text { 4. }+\mathrm{dP} / \mathrm{dtmax} \\
\text { 5. }-\mathrm{dP} / \mathrm{dtmax} \\
\text { 6. LDH } \\
\text { 7. } \mathrm{BCl}-2 \\
\text { 8. } \mathrm{Bax}\end{array}$ & $\begin{array}{l}\text { 1. } P<0.05 \\
\text { 2. } P>0.05 \\
\text { 3. } P<0.05 \\
\text { 4. } P<0.05 \\
\text { 5. } P<0.05 \\
\text { 6. } P<0.01 \\
\text { 7. } P<0.05 \\
\text { 8. } P<0.05\end{array}$ \\
\hline
\end{tabular}


TABLE 1 | Continued

\begin{tabular}{|c|c|c|c|c|c|c|c|c|}
\hline Study (years) & $\begin{array}{l}\text { Species (Sex, } n \\
=\text { experimental/ } \\
\text { control group) }\end{array}$ & Weight & Model (method) & Anesthetic & $\begin{array}{l}\text { Treatment group } \\
\text { (method to GB) }\end{array}$ & Control group & Outcome index (time) & $\begin{array}{l}\text { Intergroup } \\
\text { differences }\end{array}$ \\
\hline $\begin{array}{l}\text { Liebgott et al., } \\
2000 \text { (15) }\end{array}$ & $\begin{array}{l}\text { Wistar rats } \\
\text { (male, 12/13) }\end{array}$ & $300-350 \mathrm{~g}$ & $\begin{array}{l}\text { Langendorff Model } \\
30 \text { min } \\
\text { stabilization, } \\
\text { low-flow } \\
\text { ischemia///R (10 } \\
\mathrm{min} / 30 \\
\mathrm{~min} / 60 \mathrm{~min} \text { ) }\end{array}$ & $\begin{array}{l}\text { Pentobarbital sodium } \\
(50 \mathrm{mg} / \mathrm{kg})\end{array}$ & $\begin{array}{l}\mathrm{GB}(0.05 \mu \mathrm{g} / \mathrm{ml}) \text {, last } 10 \mathrm{~min} \text { of } \\
\text { perfusion, entire period of low-flow } \\
\text { ischemia and the first } 10 \mathrm{~min} \text { of } \\
\text { reperfusion }\end{array}$ & No treatment & $\begin{array}{l}\text { 1. LVDP } \\
\text { 2. LVEDP } \\
\text { 3. RPP } \\
\text { 4. LVdP/dt } \\
\text { 5. CF }\end{array}$ & $\begin{array}{l}\text { 1. } P<0.02 \\
\text { 2. } P<0.02 \\
\text { 3. } P<0.02 \\
\text { 4. } P<0.02 \\
\text { 5. } P<0.02\end{array}$ \\
\hline $\begin{array}{l}\text { Zhao et al., } 2014 \\
\text { (16) }\end{array}$ & $\begin{array}{l}\text { SD rats } \\
\text { (male/female, } \\
\text { 10/10) }\end{array}$ & $200-250 \mathrm{~g}$ & $\begin{array}{l}\text { Langendorff Model } \\
30 \mathrm{~min} \text { stabilization } \\
\text { I/R (20 } \mathrm{min} / 40 \mathrm{~min})\end{array}$ & $10 \%$ chloral hydrate & $\begin{array}{l}\text { GB }(10 \mu \mathrm{mol} / \mathrm{L}) \text {, for } 10 \mathrm{~min} \text { before } \\
\text { ischemia }\end{array}$ & No treatment & $\begin{array}{l}\text { 1. } \Delta \text { LVP (LVSP -LVEDP) } \\
\text { 2. }+\mathrm{dP} / \mathrm{dtmax} \\
\text { 3. }-\mathrm{dP} / \mathrm{dtmax} \\
\text { 4. } \mathrm{LDH}\end{array}$ & $\begin{array}{l}\text { 1. } P<0.01 \\
\text { 2. } P<0.05 \\
\text { 3. } P<0.01 \\
\text { 4. } P<0.01\end{array}$ \\
\hline $\begin{array}{l}\text { Zhang et al., } 2005 \\
\text { (17) }\end{array}$ & $\begin{array}{l}\text { Wistar rats } \\
\text { (male/female, 8/8) }\end{array}$ & $200-250 \mathrm{~g}$ & $\begin{array}{l}\text { Langendorff Model } \\
30 \mathrm{~min} \\
\text { stabilization, I/R } \\
\text { (15 } \mathrm{min} / 30 \mathrm{~min})\end{array}$ & $\begin{array}{l}20 \% \text { Urethane }(1.0 \\
\mathrm{g} / \mathrm{kg})\end{array}$ & $\begin{array}{l}\text { GB }(10 \mu \mathrm{mol} / \mathrm{L}) \text {, for } 10 \mathrm{~min} \text { before } \\
\text { ischemia }\end{array}$ & No treatment & $\begin{array}{l}\text { 1. Arrhythmia score } \\
\text { 2. LDH } \\
\text { 3. CK } \\
\text { 4. SOD } \\
\text { 5. MDA }\end{array}$ & $\begin{array}{l}\text { 1. } P<0.01 \\
\text { 2. } P<0.05 \\
\text { 3. } P<0.05 \\
\text { 4. } P<0.05 \\
\text { 5. } P<0.01\end{array}$ \\
\hline $\begin{array}{l}\text { Billottet et al., } \\
2005 \text { (18) }\end{array}$ & $\begin{array}{l}\text { Wistar rats } \\
\text { (male, 12/12) }\end{array}$ & $300-350 \mathrm{~g}$ & $\begin{array}{l}\text { Langendorff Model } \\
30 \text { min } \\
\text { stabilization, } \\
\text { low-flow } \\
\text { ischemia///R (20 } \\
\mathrm{min} / 20 \\
\mathrm{~min} / 60 \mathrm{~min})\end{array}$ & $\begin{array}{l}\text { Pentobarbital sodium } \\
(50 \mathrm{mg} / \mathrm{kg})\end{array}$ & $\begin{array}{l}\mathrm{GB}(0.05 \mu \mathrm{g} / \mathrm{ml}) \text { ) last } 10 \mathrm{~min} \text { of } \\
\text { perfusion, entire period of low-flow } \\
\text { ischemia and the first } 10 \mathrm{~min} \text { of } \\
\text { reperfusion }\end{array}$ & No treatment & $\begin{array}{l}\text { 1. LVDP } \\
\text { 2. LVEDP } \\
\text { 3. RPP } \\
\text { 4. LVdP/dt } \\
\text { 5. CF }\end{array}$ & $\begin{array}{l}\text { 1. } P<0.05 \\
\text { 2. } P<0.05 \\
\text { 3. } P<0.05 \\
\text { 4. } P<0.05 \\
\text { 5. } P<0.05\end{array}$ \\
\hline $\begin{array}{l}\text { Zhang et al., } 2018 \\
\text { (19) }\end{array}$ & $\begin{array}{l}\text { SD rats } \\
\text { (male, 8/8) }\end{array}$ & $250-300 \mathrm{~g}$ & $\begin{array}{l}\text { Block LAD for } \\
40 \text { min then } \\
\text { reperfusion for } 2 \mathrm{~h}\end{array}$ & $\begin{array}{l}\text { Chloral hydrate ( } 300 \\
\mathrm{mg} / \mathrm{kg})\end{array}$ & $\begin{array}{l}\text { Intraperitoneal injection GB ( } 32 \\
\left.\mathrm{mg} / \mathrm{kg}^{*} \mathrm{~d}\right) \text {, once a day, for } 7 \text { days, } \\
\text { before establishing model }\end{array}$ & $\begin{array}{l}\text { Intraperitoneal injection isasteric } \\
\text { normal saline, once a day, for } 7 \\
\text { days, after establishing model }\end{array}$ & $\begin{array}{l}\text { 1. Infarct size (AAI/AAR) } \\
\text { 2. histopathological scores } \\
\text { 3. PMNs } \\
\text { 4. TNF- } \alpha \text {, } \\
\text { 5. IL-6 } \\
\text { 6. MPO }\end{array}$ & $\begin{array}{l}\text { 1. } P<0.05 \\
\text { 2. } P<0.05 \\
\text { 3. } P<0.05 \\
\text { 4. } P<0.05 \\
\text { 5. } P<0.05 \\
\text { 6. } P<0.05\end{array}$ \\
\hline
\end{tabular}

AAl, Area at infarct; $A A R$, area at risk; AST, aspartate transaminase; Bax, BCl-2-Associated X; BCl-2, B-cell lymphoma-2; CAT, catalase; CK, creatine kinase; EF, ejection fraction; ErK, extracellular signal-regulated kinase; FS, fractional shorting; GSH-PX, glutathione peroxidase; HR, heart rate; IL-6, interleukin-6; JNK, c-Jun N-terminal kinase; LAD, left anterior descending coronary artery; LVA, left ventricular area; LVDP, left ventricular developed pressure; LVEDP, left ventricular end diastolic pressure; LDH, lactate dehydrogenase; LVIDd, left ventricular internal diameter at end-diastole; LVIDs, left ventricular internal diameter at end-systole; LVSP, Left ventricular systolic pressure; MDA, malondialdehyde; MPO, myeloperoxidase; $\pm d p / d t m a x$, Maximal rate of the increase/decrease of left ventricular pressure; p38MAPK, p38 mitogen-activated protein kinase; NF-kB, Nuclear factor-kappa B; PMNs, polymorphonuclears; RPP, rate pressure pruduct; SOD, superoxide dismutase; SV, stroke volume; SD rats, Sprague-Dawley; TNF- $\alpha$, tumor necrosis factor- $\alpha$. 
et al., 2017; Zhang et al., 2018); ejection fraction (EF) in 2 studies (Xiong and Wei, 2015; Meng, 2017), fractional shorting (FS) in 2 studies (Xiong and Wei, 2015; Meng, 2017); Maximal rate of the increase/decrease of left ventricular pressure $( \pm \mathrm{dp} / \mathrm{dtmax})$ in 8 studies (Jiao et al., 2002; Hao et al., 2009, 2013, 2016; Zhao et al., 2014; Pei et al., 2015; Hao and Dong, 2017; Yang et al., 2018); left ventricular developed pressure (LVDP) in 3 studies (Liebgott et al., 2000; Billottet et al., 2005; Yang et al., 2018); left ventricular end diastolic pressure (LVEDP) in 4 studies (Liebgott et al., 2000; Billottet et al., 2005; Hao et al., 2009, 2016); myocardial cell apoptosis index (AI) in 4 studies (Jiao et al., 2002; Bai et al., 2016; Meng, 2017; Zhuang et al., 2017); arrhythmia score in 3 studies (Zhang et al., 2005; Pei et al., 2015; Chai et al., 2018). Level of lactate dehydrogenase (LDH) was reported in 9 studies (Hao et al., 2009, 2013, 2016; Zhao et al., 2014; Pei et al., 2015; Bai et al., 2016; Cao et al., 2016; Hao and Dong, 2017; Meng, 2017); creatine kinase (CK) in 4 studies (Zhang et al., 2005; Bai et al., 2016; Cao et al., 2016; Meng, 2017) superoxide dismutase (SOD) in 5 studies (Zhang et al., 2005; Hao et al., 2013; Bai et al., 2016; Cao et al., 2016; Chai et al., 2018); malondialdehyde (MDA) in 4 studies (Zhang et al., 2005; Bai et al., 2016; Cao et al., 2016; Chai et al., 2018); glutathione peroxidase (GSH-Px) in 2 studies (Bai et al., 2016; Chai et al., 2018); caspase-3 in 2 studies (Zhuang et al., 2017; Yang et al., 2018); B-cell lymphoma-2 (Bcl2) in 4 studies (Hao et al., 2009, 2016; Xiong and Wei, 2015; Zhuang et al., 2017) and Bcl-2-Associated X (Bax) in 4 studies (Hao et al., 2009, 2016; Xiong and Wei, 2015; Zhuang et al., 2017); aspartate transaminase (AST) in 2 studies (Bai et al., 2016; Meng, 2017). Nuclear factor-kappaB (NF-kB) was mentioned in 2 study (Bai et al., 2016; Zhang et al., 2018); p38-mitogen-activated protein kinase (p38-MAPK), extracellular signal-regulated kinase (ERK) and c-Jun N-terminal kinase (JNK) in 1 study (Xiong and Wei, 2015); coronary flow (CF) in 1 study (Billottet et al., 2005); segment shortening in 1 study (Rioufol et al., 2003), mean arterial pressure (MAP) in 1 study (Rioufol et al., 2003), and tumor necrosis factor- $\alpha$ (TNF- $\alpha$ ) and interleukin-6 (IL-6) in 1 study (Zhang et al., 2018). The characteristics of selected study is provided in Table $\mathbf{1}$.

\section{Study Quality}

Detailed results of methodological quality are presented in Table 2 and Figure 3. All studies were peer-reviewed. Use of anesthetic without significant intrinsic cardioprotective activity were all described. Eleven studies (Liebgott et al., 2000; Rioufol et al., 2003; Billottet et al., 2005; Hao et al., 2009; Pei et al., 2015; Xiong and Wei, 2015; Bai et al., 2016; Zhuang et al., 2017; Chai et al., 2018; Yang et al., 2018; Zhang et al., 2018) reported control of temperature. None of the studies discussed allocation concealment, blinded assessment of outcome and the sample size calculation. No study choose the appropriate animal model. Compliance with animal welfare regulations was stated in 12 studies (Liebgott et al., 2000; Rioufol et al., 2003; Billottet et al., 2005; Zhao et al., 2014; Pei et al., 2015; Xiong and Wei, 2015; Bai et al., 2016; Hao et al., 2016; Zhuang et al., 2017; Chai et al., 2018; Yang et al., 2018; Zhang et al., 2018) and statement of potential conflict of interests was declared in 2 studies (Pei et al., 2015; Zhang et al., 2018).
TABLE 2 | Risk of bias of the included studies.

\begin{tabular}{|c|c|c|c|c|c|c|c|c|c|c|c|}
\hline Study & A & B & C & D & E & $F$ & G & $\mathbf{H}$ & I & $\mathbf{J}$ & Total \\
\hline $\begin{array}{l}\text { Zhuang et al., } \\
2017 \text { (1) }\end{array}$ & + & + & + & - & - & + & - & - & + & - & 5 \\
\hline $\begin{array}{l}\text { Xiong and } \\
\text { Wei, } 2015 \text { (2) }\end{array}$ & + & + & + & - & - & + & - & - & + & - & 5 \\
\hline $\begin{array}{l}\text { Cao et al., } \\
2016 \text { (3) }\end{array}$ & + & - & + & - & - & + & - & - & - & - & 3 \\
\hline $\begin{array}{l}\text { Meng, } 2017 \\
\text { (4) }\end{array}$ & + & - & + & - & - & + & - & - & - & - & 3 \\
\hline $\begin{array}{l}\text { Bai et al., } \\
2016 \text { (5) }\end{array}$ & + & + & + & - & - & + & - & - & + & - & 5 \\
\hline $\begin{array}{l}\text { Chai et al., } \\
2018 \text { (6) }\end{array}$ & + & + & + & - & - & + & - & - & + & - & 5 \\
\hline $\begin{array}{l}\text { Yang et al., } \\
2018 \text { (7) }\end{array}$ & + & + & + & - & - & + & - & - & + & - & 5 \\
\hline $\begin{array}{l}\text { Jiao et al., } \\
2002 \text { (8) }\end{array}$ & + & - & + & - & - & + & - & - & + & - & 3 \\
\hline $\begin{array}{l}\text { Rioufol et al., } \\
2003 \text { (9) }\end{array}$ & + & + & + & - & - & + & - & - & + & - & 5 \\
\hline $\begin{array}{l}\text { Pei et al., } \\
2015 \text { (10) }\end{array}$ & + & + & + & - & - & + & - & - & + & + & 6 \\
\hline $\begin{array}{l}\text { Hao et al., } \\
2009 \text { (11) }\end{array}$ & + & + & + & - & - & + & - & - & + & - & 4 \\
\hline $\begin{array}{l}\text { Hao and } \\
\text { Dong, } 2017 \\
\text { (12) }\end{array}$ & + & - & + & - & - & + & - & - & + & - & 3 \\
\hline $\begin{array}{l}\text { Hao et al., } \\
2013 \text { (13) }\end{array}$ & + & - & + & - & - & + & - & - & + & - & 3 \\
\hline $\begin{array}{l}\text { Hao et al., } \\
2016 \text { (14) }\end{array}$ & + & - & + & - & - & + & - & - & + & - & 4 \\
\hline $\begin{array}{l}\text { Liebgott et al., } \\
2000 \text { (15) }\end{array}$ & + & + & + & - & - & + & - & - & + & - & 5 \\
\hline $\begin{array}{l}\text { Zhao et al., } \\
2014 \text { (16) }\end{array}$ & + & - & + & - & - & + & - & - & + & - & 4 \\
\hline $\begin{array}{l}\text { Zhang et al., } \\
2005 \text { (17) }\end{array}$ & + & - & + & - & - & + & - & - & + & - & 3 \\
\hline $\begin{array}{l}\text { Billottet et al., } \\
2005 \text { (18) }\end{array}$ & + & + & + & - & - & + & - & - & + & + & 6 \\
\hline $\begin{array}{l}\text { Zhang et al., } \\
2018 \text { (19) }\end{array}$ & + & + & + & - & - & + & - & - & + & - & 5 \\
\hline
\end{tabular}

Studies fulfiling the criteria of: A, peer reviewed publication; B, control of temperature; $C$, random allocation to treatment or control; $D$, blinded induction of model; $E$, blinded assessment of outcome; $F$, use of anesthetic without significant intrinsic cardioprotective activity; G, appropriate animal model (aged, diabetic, or hypertensive); $H$, sample size calculation; I, compliance with animal welfare regulations; J, statement of potential conflict of interests.

\section{Effectiveness}

\section{Primary Outcome Measures Myocardial infarction size}

We divided the studies into two parts by different calculation methods: (1) Area at infarct/area at risk (AAI/AAR): metaanalysis of 3 studies (Jiao et al., 2002; Xiong and Wei, 2015; Zhang et al., 2018) indicated that MI size was significantly reduced using GB compared with control (SMD $-3.66,95 \% \mathrm{CI}[-4.57$ to $-2.76], P<0.00001 ; I^{2}=24 \%$ ) (Figure 4); (2) Area at infarct/left ventricular area (AAI/LVA): meta-analysis of 6 studies (Hao et al., 2009, 2016; Bai et al., 2016; Cao et al., 2016; Meng, 2017; Zhuang et al., 2017) indicated that MI size was significantly reduced by 
using GB compared with control (SMD -2.52 , 95\% CI $[-3.01$ to -2.04$\left.], P<0.00001 ; I^{2}=89 \%\right)$. There was high statistical heterogeneity among comparison of MI size (AAI/LVA). We performed a sensitivity analysis, the result remained after excluding each of these studies. In addition, subgroup analysis is layered with the following groups: experimental model (in vivo or ex vivo), stage of GB administration (pre-treatment and posttreatment), and frequency of drug administration. GB prevented MI size more obviously at the model in vivo than the model ex vivo ( $\mathrm{SMD}=-2.91$ vs. $\mathrm{SMD}=-0.98, P<0.05$, Figure 5A). Groups with more times $(\geq 5)$ of administration of GB showed a better outcome than that with fewer times $(<5)(\mathrm{SMD}=-4.13$ vs. $\mathrm{SMD}=-1.23, P<0.05$, Figure 5B). Compared with giving GB after the model, the GB pre-treatment (before introducing the model) did not produce a better outcome (SMD $=-1.37 \mathrm{vs.} \mathrm{SMD}$ $=-3.00, P<0.05$, Figure 5C).

\section{Cardiac function and the level of ST-segment elevation}

GB was significant for improving EF and FS $(P<0.05)$ (Xiong and Wei, 2015; Meng, 2017); increasing +dp/dtmax and LVSP (Jiao et al., 2002; Hao et al., 2009, 2013, 2016; Zhao et al., 2014; Pei et al., 2015; Hao and Dong, 2017; Yang et al., 2018) in systolic function $(P<0.05)$; increasing -dp/dtmax (Jiao et al., 2002; Hao et al., 2009, 2013, 2016; Zhao et al., 2014; Pei et al., 2015; Hao and Dong, 2017; Yang et al., 2018) and LVDP (Liebgott et al., 2000; Billottet et al., 2005; Yang et al., 2018) $(P<0.05)$, and decreasing LVEDP (SMD-1.98, 95\% CI [ -2.53 to -1.42$], P<0.00001 ; I^{2}=$ $43 \%$ ) (Figure 6) in diastolic function; decreasing the arrhythmia score (Zhang et al., 2005; Pei et al., 2015; Chai et al., 2018) $(P<$ $0.05)$; and reducing the ST segment elevation (Chai et al., 2018) $(P<005)$, when compared with control.

\section{Myocardial injury marker}

Meta-analysis of 4 studies (Zhang et al., 2005; Bai et al., 2016; Cao et al., 2016; Meng, 2017) indicated GB was significant for decreasing the level of CK using (SMD -1.05, 95\% CI $[-1.49$ to -0.61 ], $P<0.00001 ; I^{2}=37 \%$ ) (Figure 7A); and 2 studies (Bai et al., 2016; Meng, 2017) for decreasing the level of AST (SMD $-1.92,95 \%$ CI $[-2.55$ to -1.29$\left.], P<0.00001 ; I^{2}=0 \%\right)$ (Figure 7B) compared with control. Level of LDH was measured in 9 studies (Hao et al., 2009, 2013, 2016; Zhao et al., 2014; Pei et al., 2015; Bai et al., 2016; Cao et al., 2016; Hao and Dong, 2017; Meng, 2017), and all of them indicated GB was significant for reducing the level of $\mathrm{LDH}(P<0.05)$ compared with control.

\section{Secondary Outcome Measures}

Four studies (Jiao et al., 2002; Bai et al., 2016; Meng, 2017; Zhuang et al., 2017) showed that GB-treated group was superior to the untreated group according to the reduced AI $(n=56$, SMD -3.54 , 95\% CI [-4.17 to -2.92$\left.], P<0.00001 ; I^{2}=0 \%\right)$ (Figure 8A); 5 studies (Zhang et al., 2005; Hao et al., 2013; Bai et al., 2016; Cao et al., 2016; Chai et al., 2018) increasing SOD (SMD -2.23 , 95\% CI [1.77 to 2.69], $P<0.00001 ; I^{2}=$ 43\%) (Figure 8B); 2 studies (Bai et al., 2016; Chai et al., 2018) increasing GSH-Px (SMD 1.89, 95\% CI [1.35 to 2.43], $P<$ 0.00001; $I^{2}=0 \%$ ) (Figure 8C); 4 studies (Zhang et al., 2005; Bai et al., 2016; Cao et al., 2016; Chai et al., 2018) reducing MDA ( $P$
$<0.05) ; 2$ studies (Zhuang et al., 2017; Yang et al., 2018) reducing caspase-3 $(P<0.05)$; 4 studies (Hao et al., 2009, 2016; Xiong and Wei, 2015; Zhuang et al., 2017) increasing the ratio of Bcl2/Bax protein $(P<0.05)$; 1 study (Hao et al., 2016) reducing expression level of p-Akt $(P<0.05)$; 1 study (Chai et al., 2018) decreasing content of $\mathrm{Ca}^{2+}$ in myocardium $(P<0.05) ; 2$ studies (Bai et al., 2016; Zhang et al., 2018) decreasing expression of NF$\kappa \mathrm{B} ; 1$ study (Xiong and Wei, 2015) decreasing the level of $\mathrm{p}$-ErK, p-JNK, p-p38MAPK; 1 study (Zhang et al., 2018) suppressing PMNs infiltration $(P<0.05)$; and 1 study (Zhang et al., 2018) decreasing TNF- $\alpha$ and IL-6 $(P<0.05)$.

\section{DISCUSSION}

\section{Summary of Evidence}

In the present study, 19 studies with total 437 animals were included. GB exerted potential cardioprotective function, largely through antioxidant, anti-inflammation, anti-apoptosis and improvement of energy metabolism.

\section{Possible Mechanism}

In the early phase of reperfusion, the ischemic myocardium generates oxidative stress. Oxidative stress can increases the release of oxidative free radicals production such as ROS, RNS, and $\mathrm{OH}$ and decreases the ability of free radicals scavenging, which further leads to cardiomyocyte cell injury (NavarroYepes et al., 2014). The excessive production of oxidative free radicals in the cellular and subcellular levels peroxidates the lipid, opens the mitochondrial permeability transition pore and dysfunctions the sarcoplasmic reticulum, resulting in overloaded intracellular $\mathrm{Ca}^{2+}$ and damaged cell membrane (Hausenloy and Yellon, 2013). Therefore, decreasing the accumulation of oxygen free radicals is an important approach to protect against myocardial I/R injury. The present study showed that GB could increase the level of antioxidant enzymes such as SOD, GSH$\mathrm{Px}$ and CAT, maintain the dynamic balance of oxygen free radicals formation, restrain trigger of lipid peroxidation, protect biomembranes' integrity and function, and decrease the release of MDA from mitochondria, thereby exerts cardioprotective effect in through antioxidation.

Myocardial I/R damages the myocardium through inflammatory response (Marchant et al., 2012). During the processes of I/R injury, NF- $\mathrm{B}$ pathway (Wang et al., 2007), which is critical to the inflammation and apoptosis during various diseases, mediates the upregulated expression of various pro-inflammatory cytokines such as TNF- $\alpha$, IL-6, and IL-1 $\beta$ (Ma et al., 2014). In addition, the upstream factors of NF-kB-mitogen-activated protein kinase (MAPK) signaling pathway, including p38 MAPK, ERK, and JNK, also participates in regulating the inflammation (Chung et al., 2012; Himaya et al., 2012). The present study showed that GB exerted antiinflammatory effect via significantly inhibiting the expression of NF- $\kappa \mathrm{B}$ proteins, reducing the level of phosphorylation of $\mathrm{p} 38$, Jnk, and Erk protein, and decreasing the expression of TNF-a and IL-6 compared with control.

Myocardial I/R sequentially induce the cardiomyocyte $\mathrm{Ca}^{2+}$ overload, oxidative stress, opening of the mitochondrial 


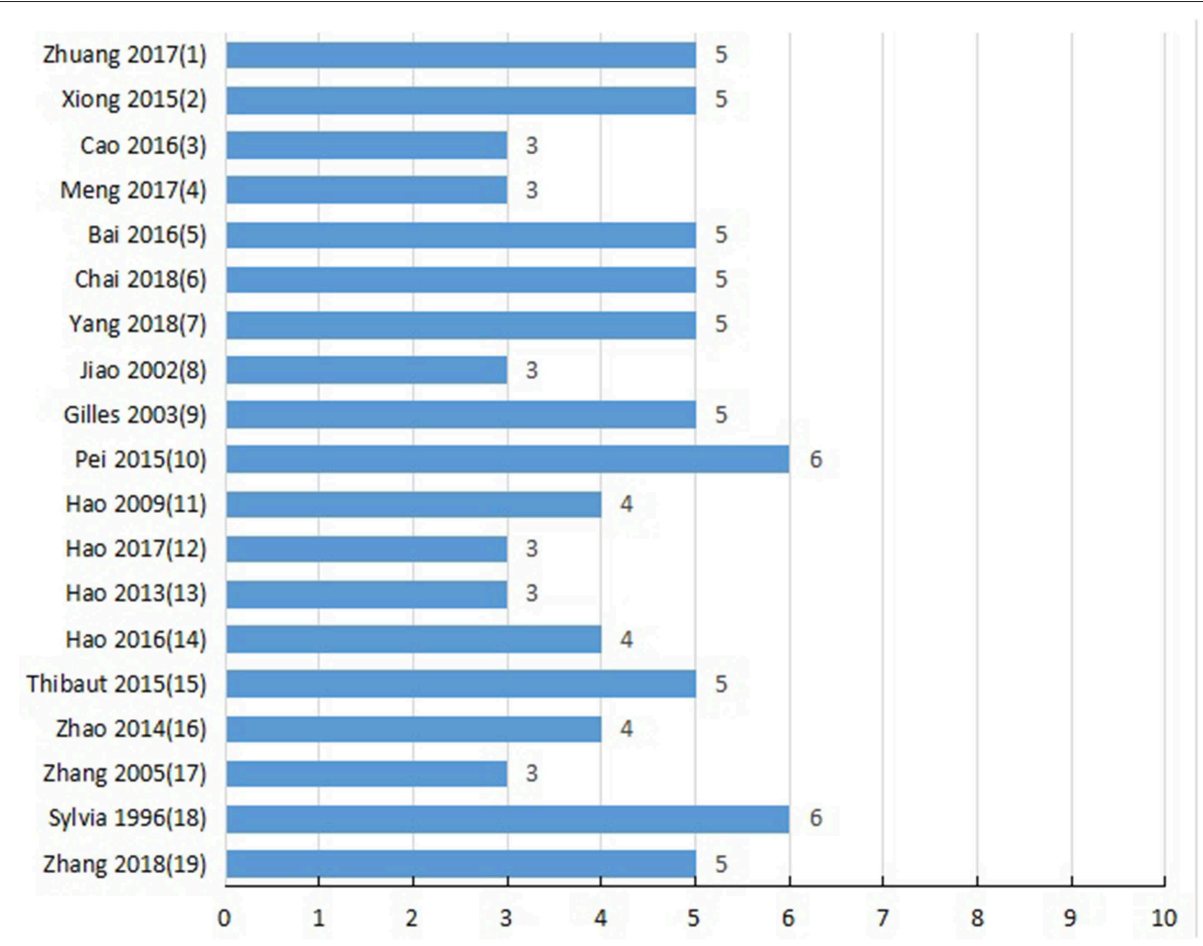

FIGURE 3 | Methodological quality for each study included.

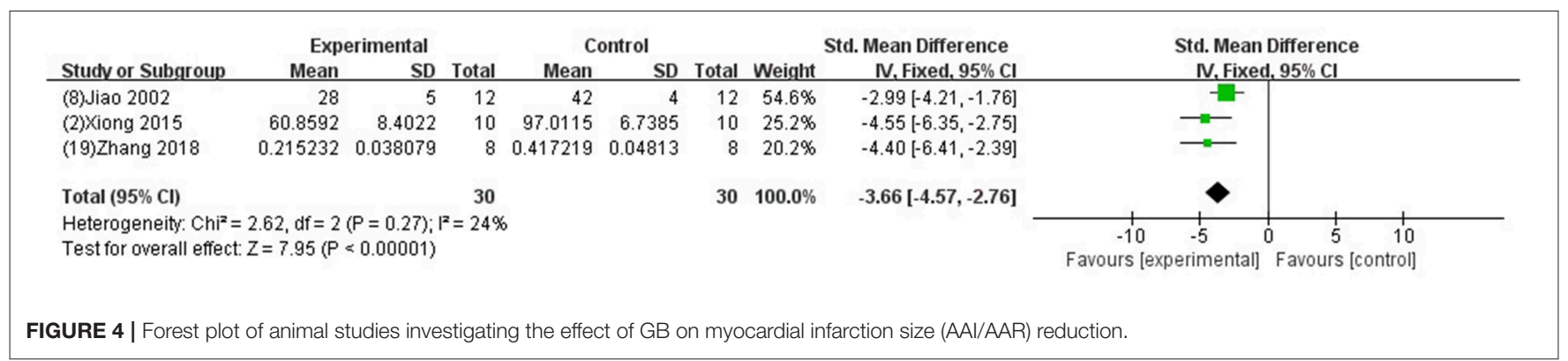

permeability transition pore, mitochondrial membrane depolarization and uncoupling of oxidative phosphorylation, and ATP depletion and cell death (Hausenloy and Yellon, 2003; Murphy and Steenbergen, 2008). The present study found that GB could improve the activity of $\mathrm{Na}^{+}-\mathrm{K}^{+}$-ATPase and $\mathrm{Ca}^{2+}-\mathrm{Mg}^{2+}$-ATPase, decrease the content of $\mathrm{Ca}^{2+}$ in myocardium, protect mitochondrial respiratory activity, reduce the accumulation of free fatty acid, lactic acid, elevate the ratio of ATP/AMP, maintain the homeostasis, and improve amplitude of cardiomyocyte shortening by modulating cardiac $\mathrm{Ca}^{2+}$ regulatory proteins such as SERCA2a, phospholamban, and $\mathrm{Na}^{+}-\mathrm{Ca}^{2+}$ exchanger.

Myocardial I/R injury resulted in cardiomyocyte apoptosis, which contributed to infarct size and ventricular dysfunction (Movassagh and Foo, 2008). The Bcl-2 family proteins were related to regulating the outer mitochondrial membrane (OMM) permeability. Increasing of OMM permeability caused by Bax could induce apoptosis, whereas decreasing the permeability caused by Bcl-2 could inhibit apoptosis (Ulgen et al., 2011). The present study indicated that GB could increase the expression of $\mathrm{Bcl}-2$, decrease the expression of $\mathrm{Bax}$, thus upregulate the ratio of $\mathrm{Bcl}-2 / \mathrm{Bax}$ to maintain the permeability of mitochondria, to inhibit activation of caspase- 3 , to inhibit the release of cytochrome $\mathrm{C}$, to regulate intracellular free calcium concentration, and to reduce the activation and infiltration of macrophage, suggesting that GB may alleviate the myocardial I/R injury by anti-apoptosis. A summary of the cardioprotective mechanism of GB is provided in schematic (Figure 9).

\section{Limitations}

Firstly, only Chinese and English studies were included, potentially causing certain bias (Guyatt et al., 2011). Secondly, the methodological quality score was generally moderate. Items identified as the core standards of research design such as 


\section{A}

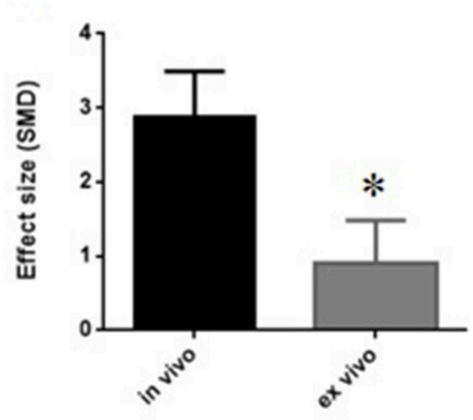

B

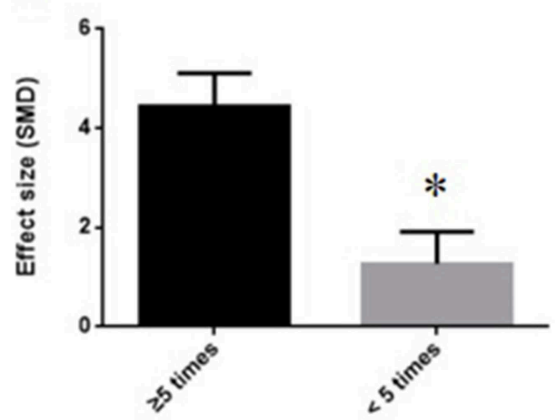

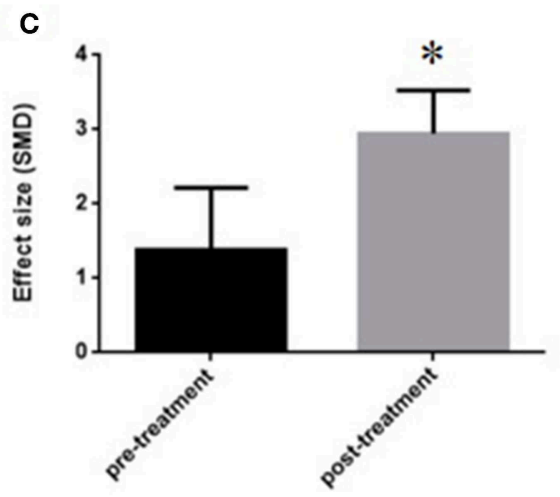

FIGURE 5 | Subgroup analyses of the myocardial infarct size (infarct area/left ventricular area). (A) Experimental model; (B) stage of GB administration; (C) frequency of drug administration. The magnitude of absolute value SMD reflected the effect size. ${ }^{*} P<0.05$ vs. control groups.

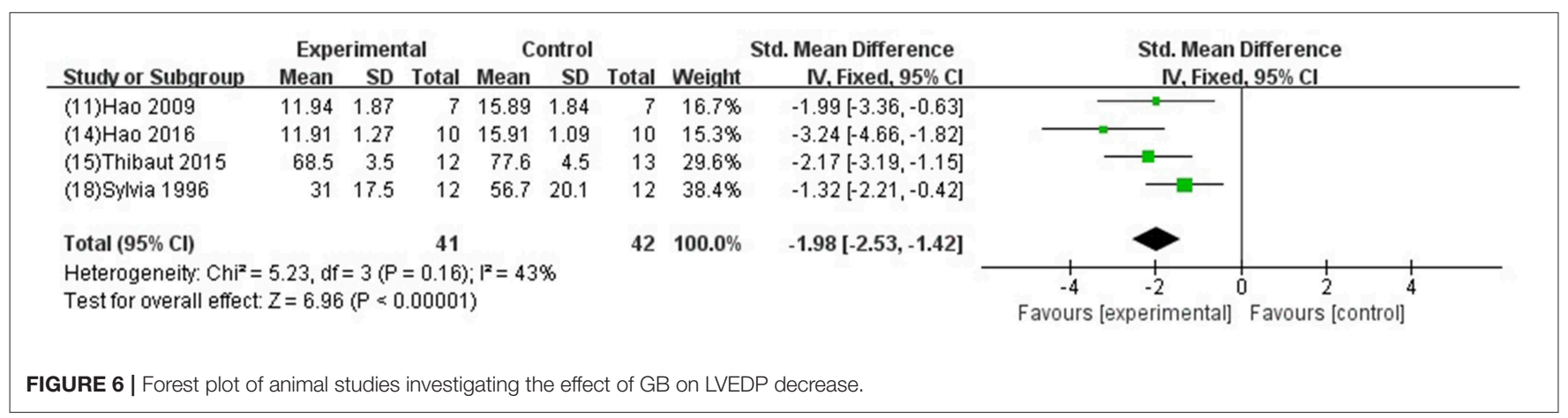

allocation concealment, blinded assessment of outcome and the sample size calculation were failed to describe. The moderate methodological quality may affect the accuracy of the results (Landis et al., 2012; Macleod et al., 2015). Finally, 4 studies (Rioufol et al., 2003; Zhang et al., 2005; Zhao et al., 2014; Cao et al., 2016) used female animals. The response and sensitivity of experimental animals with the different gender to drugs and stimuli may be different, which could influence the final experimental results (Bae and Zhang, 2005).

\section{Implications}

MI size is strongly associated with progressive worsening of left ventricular function and congestive heart failure, and enhance the long-term morbidity and mortality. Therefore, MI size can be used in clinical studies as a substitute endpoint for clinical outcomes and as an important prognostic measure when caring for patients with AMI (Ndrepepa et al., 2017). Physicians are striving to reduce the MI size so as to improve the prognosis of patients (Begley and Ioannidis, 2015). In animal studies, MI size is the robust primary outcome that invariably used to evaluate the efficacy of various pharmacological or non-pharmacological strategies that prevent I/R injury (Zhou et al., 2018). In the present study, the results showed that GB could reduce the MI size, improve the diastolic and systolic function, decrease the level of CK, AST, and LDH, increase the level of GSH-Px. Thus, our study is valuable to the translational development. 
A

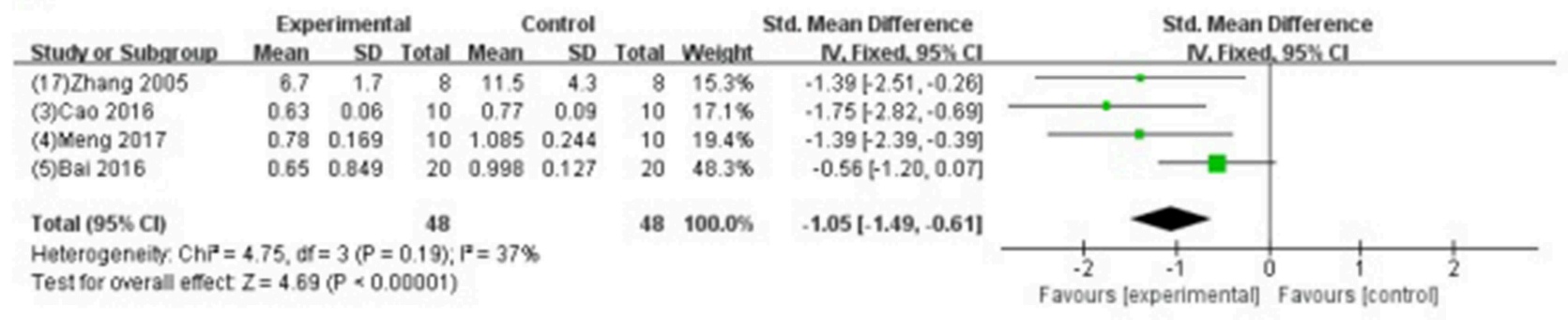

B

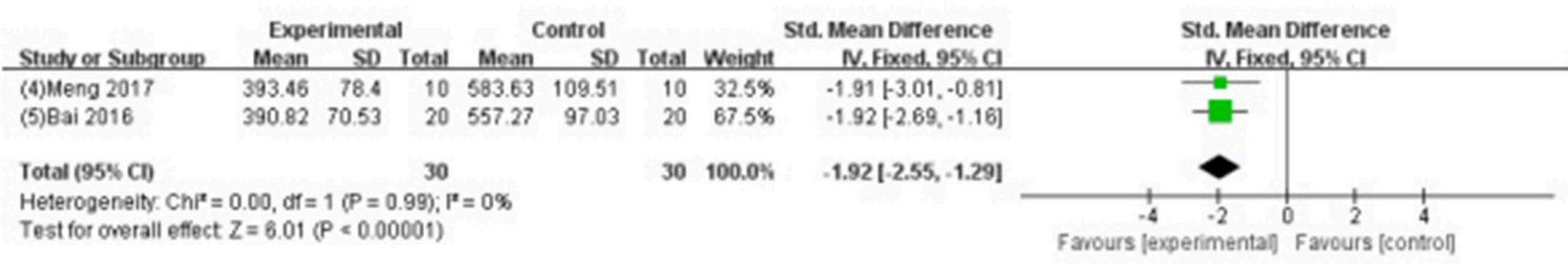

FIGURE 7 | (A) Forest plot of animal studies investigating the effect of GB on creatine kinase decrease. (B) Forest plot of animal studies investigating the effect of GB on aspartate transaminase decrease.

Animal studies are mainly targeted toward enriching our understanding of the mechanisms, etiology of human diseases, and forming the foundation on which future studies are built (Begley and Ioannidis, 2015). Accurate and complete reporting of animal studies is important to support the valid and reproducible research, while lower-quality reporting is related to deficiencies in experimental design that introduce bias and exaggerate effect sizes in the literature (Leung et al., 2018). The poor experimental design would restrain the translational development from animal studies into the clinical trials (Briel et al., 2013). The ARRIVE (Animals in Research: Reporting In Vivo Experiments) guidelines consist of a 20 -item checklist published in 2010 with the aim of promoting the transparency and accuracy of animal research reporting, and of maximizing the availability and utility of information gained from every experiment (Gulin et al., 2015; Liu et al., 2016). In the present study, owing to the methodological quality, the results should be interpreted with caution. Thus, we proposed that further experiment design and reporting of the animal studies should refer to the ARRIVE guidelines (Kilkenny et al., 2010).

Inappropriate selection of the animal models could contribute to spurious or inconsistent results, as well as unnecessary animal use (Lecour et al., 2014). Given that animals with complications or risk factors such as ageing, diabetes and hypertension are close to the patients with myocardial infarction amalgamated with hypertension, hyperlipidemia, diabetes, insulin resistance, heart failure, altered coronary circulation and aging, we advise that further studies should use the appropriate animals instead of the young and healthy ones (Ferdinandy et al., 2014). These cardiovascular risks and comorbidities need to treat both short and long term, and contribute to the development of
IR injury and complicate the therapy (Rossello and Yellon, 2016).

Animal models are often used to study the effectiveness of a drug or procedure and explore the mechanisms before proceeding to clinical trial (Roberts et al., 2002; Liao et al., 2012), which can be divided into in vivo and ex vivo models. The environment in which the in vivo myocardial infarction model located is exposed to normal humoral influences and neuronal regulation, and thus in vivo models can fully test the effect of the drug as an organic whole. However, many internal factors act as confounders, resulting in the deficiencies of the drug's effectiveness. In addition, the process of ligating the coronary artery and putting it back into the body needs high-tech requirements and high costs. In comparison, the isolated heart model with its broad spectrum of physiological, biochemical, and morphological measurements is a powerful ex vivo cardiovascular research tool that can be applied under numerous pathological condition (Skrzypiec-Spring et al., 2007; Liao et al., 2012). It possesses high-reproducibility, convenient operation, accurate results and time-saving due to the relatively simple process. Meanwhile, it is conducive to explore the mechanisms because various influencing factors can be strictly controlled easily. Compared with the cell models, the isolated heart model provides a more realistic correlation with the in vivo studies (Nahar et al., 2013). However, since the experimental environment of the ex vivo model can't fully simulate the internal environment, the results may not be comprehensive. According to the characteristics of animal models, in vivo experiments are mainly used to study the pharmacodynamics of pharmaceuticals, while the ex vivo experiments are more commonly contributed to explore mechanisms and physiological processes. This may explain the reason why the effect on infarct size in vivo heart 
A

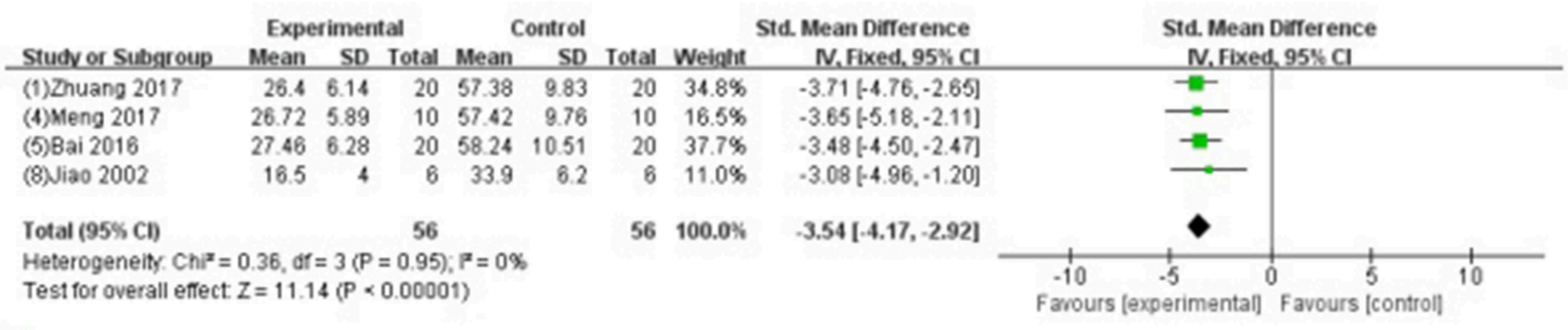

B

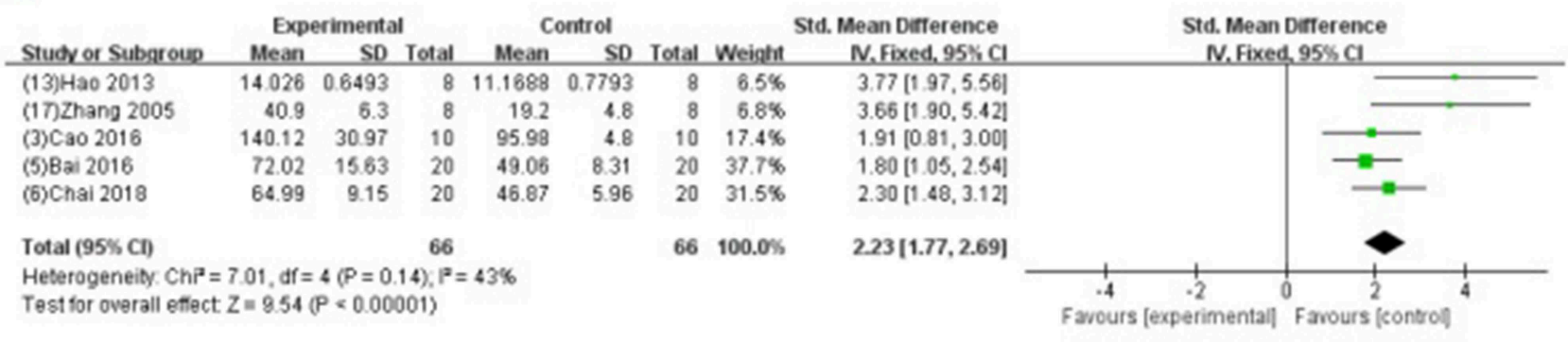

C

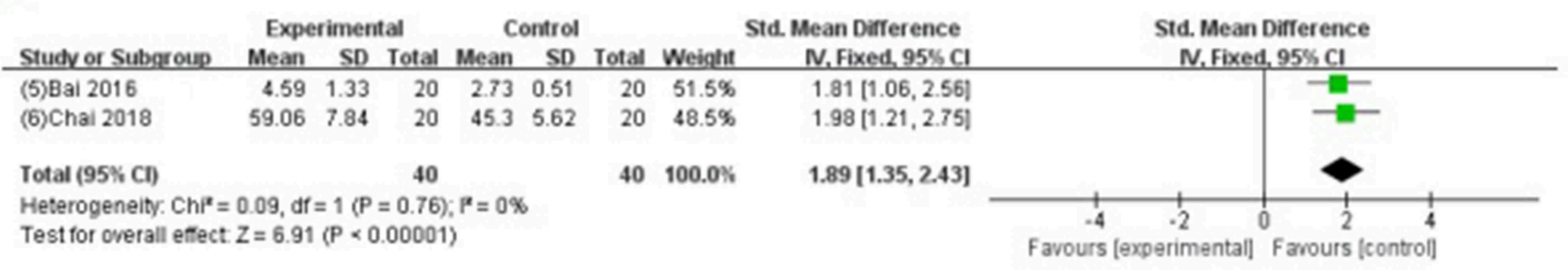

FIGURE 8 | (A) Forest plot of animal studies investigating the effect of GB onapoptosis index decrease. (B) Forest plot of animal studies investigating the effect of GB onsuperoxide dismutasedecrease. (C) Forest plot of animal studies investigating the effect of GB on glutathione peroxidase.

model (Bai et al., 2016; Cao et al., 2016; Meng, 2017; Zhuang et al., 2017) showed better effects compared with the isolated heart model in the present study (Hao et al., 2009, 2016). Future experiments can select different models for different experimental purposes. However, no single experimental model is sufficient to investigate of the mechanism and therapeutic effects of the drug simultaneously (Jiang et al., 2016). In order to test the drug research systematically and deeply, we strongly suggest to utilize two models that complement each other.

The group with more times $(\geq 5)$ of drug administration (Bai et al., 2016; Meng, 2017; Zhuang et al., 2017) showed a better outcome than that with fewer times $(<5)$ (Hao et al., 2009, 2016; Cao et al., 2016). The pharmacological effect of TCM comes from biologically active part or active chemical components, which demonstrate the preventive and therapeutic effects. The bioactive ingredients of TCM are very complex and totally different from that of modern western medicine. The research on a scientific theory about TCM's pharmacological effects and bioactive ingredients is limited (Huang et al., 2015). The bioavailability of active ingredients in TCM is low, and their affinity is weak ( $\mathrm{Yu}$ and $\mathrm{Lu}, 2018$ ). The effect of drug treatment is relatively slow, which requires a certain period of treatment. GB is a pure compound of the Ginkgo biloba leaves. In the present study, 3 studies (Bai et al., 2016; Meng, 2017; Zhuang et al., 2017) were administrated GB, qd, for 7 days, whereas other 3 studies (Hao et al., 2009, 2016; Cao et al., 2016) were administrated GB only 1 time. The drug concentration in the body increases proportionally when the dosage of the drug is administrated more times. Repeated administration of drugs would help to achieve and maintain the effective blood concentrations. Therefore, further studies administer GB for multiple times.

GB was an antagonist of PAF that inhibits platelet activation/aggregation via competitive binding to PAF receptor (Heinroth-Hoffmann et al., 1990). Owing to the slow onset time of herbal efficacy, administration of herbs before the induction of models was often used for herbal pharmacological 


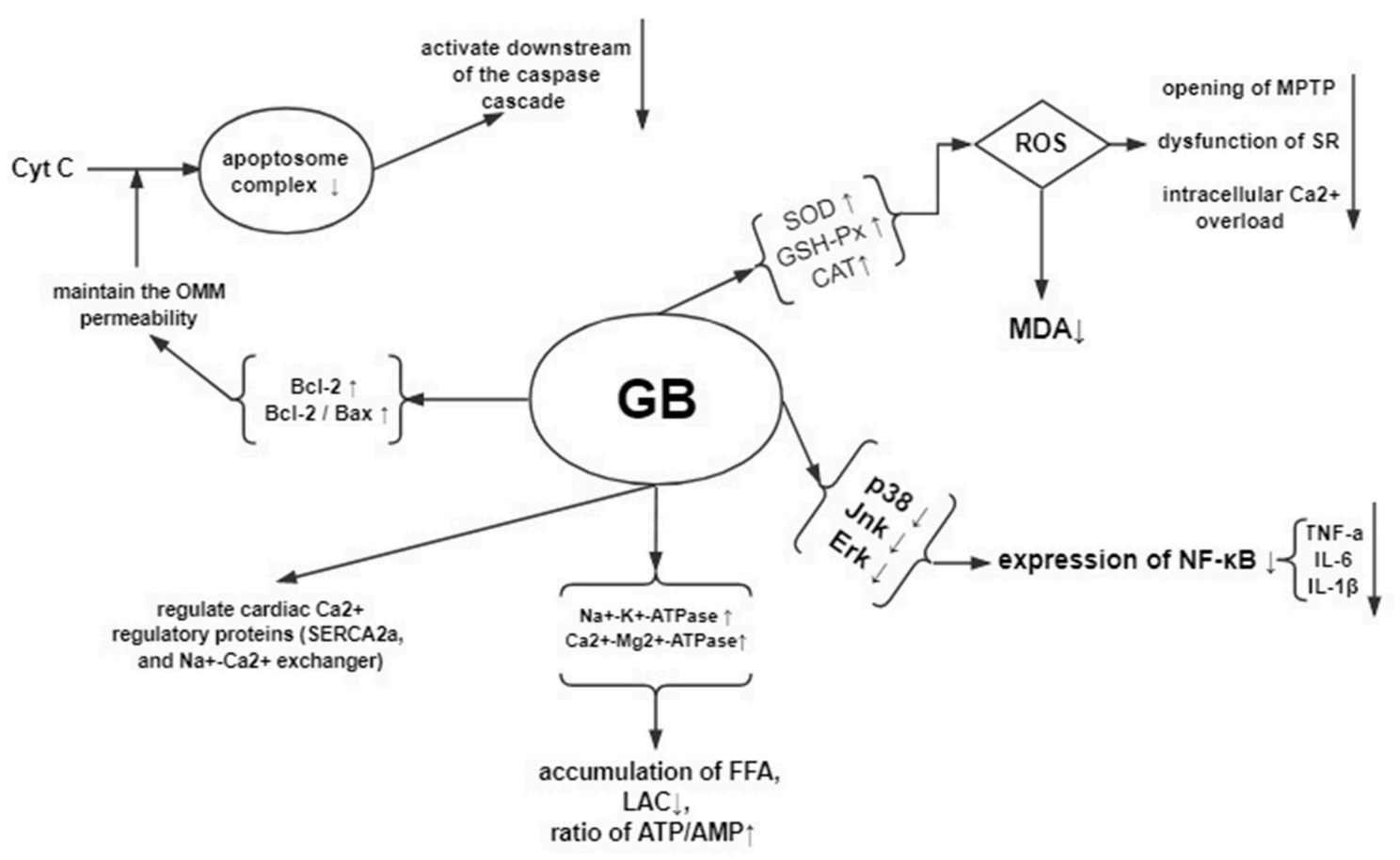

FIGURE 9 | Mechanisms of GB exerted cardioprotective effects in myocardial ischemia/reperfusion injury.

researches in order to reach the effective plasma concentration. The treatment post-model induction is more in line with clinical practice. Thus, it is more appropriate to study herbal pharmacology in both two different administrations. The results of present study showed that the efficacy in post-model induction group were better than that in the pre-model induction group, suggesting that the GB has fast onset time of decreasing platelet function directly via inhibiting the effect of PAF to alleviate myocardial I/R injury. Further study of this novel issue is needed.

Mechanisms underlying the pathogenesis of myocardial I/R injury are particularly complex, multifactorial and highly interconnected (Russo et al., 2017). The oxidative stress, apoptosis, energy metabolism disorder, and inflammation were described above. Other mechanisms such as Calcium paradox, $\mathrm{pH}$ paradox, metabolic modulation, Magnesium therapeutic, hypothermia, and autophagy, were also related to reperfusion injury. Based on the understanding of the pathophysiology of $\mathrm{I} / \mathrm{R}$ injury, several advance non-pharmacological and pharmacological cardioprotective strategies were proposed to protect the ischemic myocardium in recent years. About non-pharmacological therapy, studies in animal models showed that ischemic pre-and post-conditioning, remote ischemic conditioning and hypothermia potentially stimulate the cardioprotective response. Meanwhile, different cardioprotective pharmacological agents have been confirmed in preclinical studies. These pharmacological agents include agonists of $\beta$-adrenoceptors $(\beta$-AR) or adenosine receptors, L-type voltage-dependent $\mathrm{Ca}^{2+}$ channels (VDCC) or mitochondrial
$\mathrm{Ca}^{2+}$ uniporter (MCU) blockers, anti-platelet, modulators of $\mathrm{NO}$ biosynthesis or $\mathrm{Na}^{+} / \mathrm{Ca}^{2+}$ exchanger (NCX), resveratrol, methylene blue and intestinal lipase inhibitors (Caricati-Neto et al., 2019). In the future, nanotechnology-guided drug delivery systems will become new direction (Minamino, 2012), and novel cardioprotective mechanisms of GB should be investigated.

Assessing the risk of bias of the individual studies is a key feature of a systematic review. The Cochrane risk of bias (RoB) Tool is developed by the Cochrane Collaboration to establish consistency and avoid discrepancies in assessing the methodological quality of RCTs (Hooijmans et al., 2014). Analogously, when conducting systematic review of preclinical studies, the main difference is the methodological quality assessment methods. Some checklists have been developed to evaluate the quality of in vivo animal intervention experiments (Hooijmans et al., 2014; Zhang et al., 2017), including the SYstematic Review Centre for Laboratory animal Experimentation (SYRCLE)'s RoB tool and the Collaborative Approach to Meta-Analysis and Review of Animal Data from Experimental Studies (CAMARADES) 10-item checklist. About in vitro studies, the $\mathrm{ROB}$ assessment can refer to 10 -item scale developed by our group (Bao et al., 2018). However, there are no quality assessment tools for a systematical review of ex vivo studies. The 9 out of 10 items of CAMARADES checklist are involved in the process of ex vivo studies except an item of animal model. Therefore, it is appropriate that we choose the CAMARADES 10-item list to assess the quality of ex vivo studies. The ex vivo studies are commonly contributed to 
explore mechanisms and physiological processes; however, many experimental did not choose animal models with comorbidities such as diabetes, hypertension, and atherosclerosis. When using the CAMARADES 10-item checklist to evaluate the quality of study, the point of appropriate animal model did not award that potentially caused bias accordingly. Thus, further ex vivo studies should choose appropriate models that make the results more accurate and comprehensive so as to improve efficiency in the design of study and promote the translation of findings from animal studies into clinic.

\section{CONCLUSION}

The present study demonstrated that GB could alleviate the myocardial I/R injury, mainly through the antioxidation, anti-inflammation, anti-apoptosis, and the improvement of energy metabolism.

\section{REFERENCES}

Bae, S., and Zhang, L. (2005). Gender differences in cardioprotection against ischemia/reperfusion injury in adult rat hearts: focus on Akt and protein kinase C signaling. J. Pharmacol. Exp. Ther. 315, 1125-1135. doi: 10.1124/jpet.105.090803

Bai, Z. C., Chen, M. C., and Li, H. F. (2016). The effects of ginkgolide B on oxidative stress in rats with myocardial ischemia-reperfusion injury. JETCM 25, 1480-1483. doi: 10.3969/j.issn.1004-745X.2016.08.008

Bao, X. Y., Zheng, Q., Tong, Q., Zhu, P. C., Zhuang, Z., Zheng, G. Q., et al. (2018). Danshensu for myocardial ischemic injury: preclinical evidence and novel methodology of quality assessment tool. Front. Pharmacol. 9:1445. doi: 10.3389/fphar.2018.01445

Begley, C. G., and Ioannidis, J. P. (2015). Reproducibility in science: improving the standard for basic and preclinical research. Circ. Res. 116, 116-126. doi: 10.1161/CIRCRESAHA.114.303819

Bilia, A. R. (2002). Ginkgo biloba L. Fitoterapia 73, 276-279. doi: 10.1016/S0367-326X(02)00071-0

Billottet, L., Martel, S., Culcasi, M., Drieu, K., Carrupt, P.-A., and Pietri, S. (2005). Influence of lipophilicity and stereochemistry at the C7 position on the cardioprotective and antioxidant effect of ginkgolides during rat heart ischemia and reperfusion. Drug Dev. Res. 64, 157-171. doi: 10.1002/ddr.10424

Binder, A., Ali, A., Chawla, R., Aziz, H. A., Abbate, A., and Jovin, I. S. (2015). Myocardial protection from ischemia-reperfusion injury post coronary revascularization. Expert Rev Cardiovasc Ther. 13, 1045-1057. doi: 10.1586/14779072.2015.1070669

Briel, M., Muller, K. F., Meerpohl, J. J., Von Elm, E., Lang, B., Motschall, E., et al. (2013). Publication bias in animal research: a systematic review protocol. Syst. Rev. 2, 23. doi: $10.1186 / 2046-4053-2-23$

Bulluck, H., Yellon, D. M., and Hausenloy, D. J. (2016). Reducing myocardial infarct size: challenges and future opportunities. Heart 102, 341-348. doi: 10.1136/heartjnl-2015-307855

Cao, J. P., Li, X., Ma, Y., Li, X., Ma, Y., Shu, J. L., et al. (2016). Study of protective effects of ginkgolide B on myocardial ischemia reperfusion injury in rats. Prev. Treat. Cardio Cereb. Vasc. Dis. 16, 8-10. doi: 10.3969/j.issn.1009-816x.2016.01.03

Caricati-Neto, A., Errante, P. R., and Menezes-Rodrigues, F. S. (2019). Recent advances in pharmacological and non-pharmacological strategies of cardioprotection. Int. J. Mol. Sci. 20:E4002. doi: 10.3390/ijms201 64002

Chai, L. N., Du, R., and Zhang, Y. Y. (2018). Effects and mechnism of ginkgolide $\mathrm{B}$ on myocardial arrhythmia induced by myocardial ischemia - reperfusion. JETCM 27, 619-621. doi: 10.3969/j.issn.1004-745X.2018.04.016

\section{DATA AVAILABILITY STATEMENT}

The raw data supporting the conclusions of this manuscript will be made available by the authors, without undue reservation, to any qualified researcher.

\section{AUTHOR CONTRIBUTIONS}

$\mathrm{YW}, \mathrm{GZ}$, and P-CZ contributed to the study conception and design. P-CZ, QT, ZZ, Z-HW, and L-HD contributed to the acquisition, the analysis, and/or the interpretation of data. GZ and YW gave the final approval and contributed overall responsibility for this published work.

\section{FUNDING}

This work was supported by the grant of National Natural Science Foundation of China (81473491/81573750/81173395/H2902).

Chung, J. W., Choi, R. J., Seo, E. K., Nam, J. W., Dong, M. S., Shin, E. M., et al. (2012). Anti-inflammatory effects of (Z)-ligustilide through suppression of mitogen-activated protein kinases and nuclear factor- $\mathrm{\kappa B}$ activation pathways. Arch. Pharm. Res. 35, 723-732. doi: 10.1007/s12272-012-0417-z

Eltzschig, H. K., and Eckle, T. (2011). Ischemia and reperfusion-from mechanism to translation. Nat. Med. 17, 1391-1401. doi: 10.1038/nm.2507

Ferdinandy, P., Hausenloy, D. J., Heusch, G., Baxter, G. F., and Schulz, R. (2014). Interaction of risk factors, comorbidities, and comedications with ischemia/reperfusion injury and cardioprotection by preconditioning, postconditioning, and remote conditioning. Pharmacol. Rev. 66, 1142-1174. doi: $10.1124 /$ pr.113.008300

Frank, A., Bonney, M., Bonney, S., Weitzel, L., Koeppen, M., and Eckle, T. (2012). Myocardial ischemia reperfusion injury: from basic science to clinical bedside. Semin. Cardiothorac. Vasc. Anesth. 16, 123-132. doi: 10.1177/1089253211436350

Gulin, J. E., Rocco, D. M., and Garcia-Bournissen, F. (2015). Quality of reporting and adherence to ARRIVE guidelines in animal studies for chagas disease preclinical drug research: a systematic review. PLoS Negl. Trop. Dis. 9:e0004194. doi: 10.1371/journal.pntd.00 04194

Guyatt, G. H., Oxman, A. D., Montori, V., Vist, G., Kunz, R., Brozek, J., et al. (2011). GRADE guidelines: 5. Rating the quality of evidencepublication bias. J. Clin. Epidemiol. 64, 1277-1282. doi: 10.1016/j.jclinepi.2011. 01.011

Hao, Y. L., and Dong, W. (2017). Combination of ginkgolide A and B in improving the cardiac function in aged rats with ischemia/reperfusion injury. J. Qiqihar Univ. Med. 38, 1863-1865. doi: 10.3969/j.issn.1002-1256.2017. 16.002

Hao, Y. L., Jiang, Y. L., Wang, Y. W., Jiang, R., He, J., Yu, C., et al. (2013). Effects of combined treatment with ginkgolides $\mathrm{B}$ and $\mathrm{C}$ on rat cardiac function after ischemia/reperfusion injury. Acta Acad. Med. Xuzhou 33, 733-736.

Hao, Y. L., Sun, Y., Xu, C., Jiang, X., Sun, H., Wu, Q., et al. (2009). Improvement of contractile function in isolated cardiomyocytes from ischemia-reperfusion rats by ginkgolide B pretreatment. J. Cardiovasc. Pharmacol. 54, 3-9. doi: 10.1097/FJC.0b013e3181a91410

Hao, Y. L., Yang, Y., Wu, J. X., Peng, K., Zhang, C. P., Sun, H., et al. (2016). Improvement of contractile function of isolated hearts with ischemiareperfusion injury of aged rats by ginkgolide B post-treatment. Chin. J. New Drugs 25, 2707-2713.

Hausenloy, D. J., and Yellon, D. M. (2003). The mitochondrial permeability transition pore: its fundamental role in mediating cell death during ischaemia and reperfusion. J. Mol. Cell. Cardiol. 35, 339-341. doi: 10.1016/S0022-2828(03)00043-9 
Hausenloy, D. J., and Yellon, D. M. (2013). Myocardial ischemia-reperfusion injury: a neglected therapeutic target. J. Clin. Invest. 123, 92-100. doi: 10.1172/JCI62874

Heinroth-Hoffmann, I., Hauser, A., and Mest, H. J. (1990). Effects of platelet activating factor antagonists on arachidonic acid-induced platelet aggregation and TXA2 formation. Prostaglandins Leukot. Essent. Fatty Acids 41, 181-182. doi: 10.1016/0952-3278(90)90087-2

Himaya, S. W., Ryu, B., Qian, Z. J., and Kim, S. K. (2012). Paeonol from Hippocampus kuda Bleeler suppressed the neuro-inflammatory responses in vitro via NF- $\kappa$ B and MAPK signaling pathways. Toxicol. In Vitro 26, 878-887. doi: 10.1016/j.tiv.2012.04.022

Hooijmans, C. R., Rovers, M. M., De Vries, R. B., Leenaars, M., Ritskes-Hoitinga, M., and Langendam, M. W. (2014). SYRCLE's risk of bias tool for animal studies. BMC Med. Res. Methodol. 14:43. doi: 10.1186/1471-2288-14-43

Huang, Y., Zhao, Y., Liu, F., and Liu, S. (2015). Nano traditional Chinese medicine: current progresses and future challenges. Curr. Drug Targets 16, 1548-1562. doi: 10.2174/1389450116666150309122334

Ibanez, B., Heusch, G., Ovize, M., and Van De Werf, F. (2015). Evolving therapies for myocardial ischemia/reperfusion injury. J. Am. Coll. Cardiol. 65, 1454-1471. doi: 10.1016/j.jacc.2015.02.032

Jacobs, B. P., and Browner, W. S. (2000). Ginkgo biloba: a living fossil. Am. J. Med. 108, 341-342. doi: 10.1016/S0002-9343(00)00290-4

Jiang, D. S., Yi, X., Zhu, X. H., and Wei, X. (2016). Experimental in vivo and ex vivo models for the study of human aortic dissection: promises and challenges. Am. J. Transl. Res. 8, 5125-5140.

Jiao, X. Y., Luo, N., Zhi, J. M., and Zhao, R. R. (2002). Effects of exogenous platelet activating factor and its antagonist ginkgolide B on ischemiareperfusion injury of myocardium in rats. Chin. J. Pharmacol. Toxic. 2, 81-87. doi: 10.3321/j.issn:1000-3002.2002.02.001

Kilkenny, C., Browne, W. J., Cuthill, I. C., Emerson, M., and Altman, D. G. (2010). Improving bioscience research reporting: the ARRIVE guidelines for reporting animal research. J. Pharmacol. Pharmacother. 1, 94-99. doi: 10.4103/0976-500X.72351

Landis, S. C., Amara, S. G., Asadullah, K., Austin, C. P., Blumenstein, R., Bradley, E. W., et al. (2012). A call for transparent reporting to optimize the predictive value of preclinical research. Nature 490, 187-191. doi: 10.1038/nature11556

Lecour, S., Botker, H. E., Condorelli, G., Davidson, S. M., Garcia-Dorado, D., Engel, F. B., et al. (2014). ESC working group cellular biology of the heart: position paper: improving the preclinical assessment of novel cardioprotective therapies. Cardiovasc. Res. 104, 399-411. doi: 10.1093/cvr/ cru225

Leung, V., Rousseau-Blass, F., Beauchamp, G., and Pang, D. S. J. (2018). ARRIVE has not ARRIVEd: support for the ARRIVE (Animal Research: Reporting of in vivo Experiments) guidelines does not improve the reporting quality of papers in animal welfare, analgesia or anesthesia. PLoS ONE 13:e0197882. doi: 10.1371/journal.pone. 0197882

Li, S. J., Geng, J. L., Zhang, Y., Yang, N., Zhou, F., Akiye, J.Y., et al. (2017). Advances in pharmacological research of Ginkgo biloba. Drug Eval. Res. 40, 731-741. doi: 10.7501/j.issn.1674-6376.2017.06.002

Liao, R., Podesser, B. K., and Lim, C. C. (2012). The continuing evolution of the Langendorff and ejecting murine heart: new advances in cardiac phenotyping. Am. J. Physiol. Heart Circ. Physiol. 303, H156-H167. doi: 10.1152/ajpheart.00333.2012

Liebgott, T., Miollan, M., Berchadsky, Y., Drieu, K., Culcasi, M., and Pietri, S. (2000). Complementary cardioprotective effects of flavonoid metabolites and terpenoid constituents of Ginkgo biloba extract (EGb 761) during ischemia and reperfusion. Basic Res. Cardiol. 95, 368-377. doi: 10.1007/s0039500 70035

Liu, Y., Zhao, X., Mai, Y., Li, X., Wang, J., Chen, L., et al. (2016). Adherence to ARRIVE guidelines in Chinese journal reports on neoplasms in animals. PLoS ONE 11:e0154657. doi: 10.1371/journal.pone.0154657

Ma, L., Liu, H., Xie, Z., Yang, S., Xu, W., Hou, J., et al. (2014). Ginsenoside Rb3 protects cardiomyocytes against ischemia-reperfusion injury via the inhibition of JNK-mediated NF- $\kappa \mathrm{B}$ pathway: a mouse cardiomyocyte model. PLoS ONE 9:e103628. doi: 10.1371/journal.pone.0103628

Maclennan, K. M., Darlington, C. L., and Smith, P. F. (2002). The CNS effects of Ginkgo biloba extracts and ginkgolide B. Prog. Neurobiol. 67, 235-257. doi: 10.1016/S0301-0082(02)00015-1
Macleod, M. R., Lawson McLean, A., Kyriakopoulou, A., Serghiou, S., De Wilde, A., Sherratt, N., et al. (2015). Risk of bias in reports of in vivo research: a focus for improvement. PLoS Biol. 13:e1002273. doi: 10.1371/journal.pbio.10 02273

Macleod, M. R., O'collins, T., Howells, D. W., and Donnan, G. A. (2004), Pooling of animal experimental data reveals influence of study design and publication bias. Stroke 35, 1203-1208. doi: 10.1161/01.STR.0000125719.25 853.20

Marchant, D. J., Boyd, J. H., Lin, D. C., Granville, D. J., Garmaroudi, F. S., and Mcmanus, B. M. (2012). Inflammation in myocardial diseases. Circ. Res. 110, 126-144. doi: 10.1161/CIRCRESAHA.111.243170

Meng, J. F. (2017). Protective effects of ginkgolide $B$ on rats with myocardial ischemia reperfusion injury. JETCM 26, 1977-1979. doi: 10.3969/j.issn.1004-745X.2017.11.028

Minamino, T. (2012). Cardioprotection from ischemia/reperfusion injury: basic and translational research. Circ. J. 76, 1074-1082. doi: 10.1253/circi.CJ-12-0132

Movassagh, M., and Foo, R. S. (2008). Simplified apoptotic cascades. Heart Fail. Rev. 13, 111-119. doi: 10.1007/s10741-007-9070-x

Murphy, E., and Steenbergen, C. (2008). Mechanisms underlying acute protection from cardiac ischemia-reperfusion injury. Physiol. Rev. 88, 581-609. doi: $10.1152 /$ physrev.00024.2007

Nahar, K., Gupta, N., Gauvin, R., Absar, S., Patel, B., Gupta, V., et al. (2013). In vitro, in vivo and ex vivo models for studying particle deposition and drug absorption of inhaled pharmaceuticals. Eur. J. Pharm. Sci. 49, 805-818. doi: 10.1016/j.ejps.2013.06.004

Navarro-Yepes, J., Burns, M., Anandhan, A., Khalimonchuk, O., Del Razo, L. M., Quintanilla-Vega, B., et al. (2014). Oxidative stress, redox signaling, and autophagy: cell death versus survival. Antioxid. Redox Signal. 21, 66-85. doi: 10.1089/ars.2014.5837

Ndrepepa, G., Colleran, R., and Kastrati, A. (2017). Reperfusion injury in STsegment elevation myocardial infarction: the final frontier. Coron. Artery Dis. 28, 253-262. doi: 10.1097/MCA.0000000000000468

Pei, H. X., Hua, R., Guan, C. X., and Fang, X. (2015). Ginkgolide B reduces the degradation of membrane phospholipids to prevent ischemia/reperfusion myocardial injury in rats. Pharmacology 96, 233-239. doi: 10.1159/000438945

Rioufol, G., Pietri, S., Culcasi, M., Loufoua, J., Staat, P., Pop, C., et al. (2003). Ginkgo biloba extract EGb 761 attenuates myocardial stunning in the pig heart. Basic Res. Cardiol. 98, 59-68. doi: 10.1007/s00395-003-0380-4

Roberts, I., Kwan, I., Evans, P., and Haig, S. (2002). Does animal experimentation inform human healthcare? Observations from a systematic review of international animal experiments on fluid resuscitation. BMJ 324, 474-476. doi: $10.1136 /$ bmj.324.7335.474

Rossello, X., and Yellon, D. M. (2016). Cardioprotection: the disconnect between bench and bedside. Circulation 134, 574-575. doi: 10.1161/CIRCULATIONAHA.116.022829

Russo, I., Penna, C., Musso, T., Popara, J., Alloatti, G., Cavalot, F., et al. (2017). Platelets, diabetes and myocardial ischemia/reperfusion injury. Cardiovasc. Diabetol. 16:71. doi: 10.1186/s12933-017-0550-6

Sandercock, P., and Roberts, I. (2002). Systematic reviews of animal experiments. Lancet 360:586. doi: 10.1016/S0140-6736(02)09812-4

Skrzypiec-Spring, M., Grotthus, B., Szelag, A., and Schulz, R. (2007). Isolated heart perfusion according to Langendorff-still viable in the new millennium. J. Pharmacol. Toxicol. Methods 55, 113-126. doi: 10.1016/j.vascn.2006.05.006

Stewart, L. A., Clarke, M., Rovers, M., Riley, R. D., Simmonds, M., Stewart, G., et al. (2015). Preferred reporting items for systematic review and metaanalyses of individual participant data: the PRISMA-IPD statement. JAMA 313, 1657-1665. doi: 10.1001/jama.2015.3656

Ulgen, B. O., Field, M. G., Qureshi, W., Knight, R. A., Stephanou, A., Latchman, D. S., et al. (2011). The role of minocycline in ischemia-reperfusion injury: a comprehensive review of an old drug with new implications. Recent Pat. Cardiovasc. Drug Discov. 6, 123-132. doi: 10.2174/157489011795933783

Wang, Q., Tang, X. N., and Yenari, M. A. (2007). inflammatory response in stroke. J. Neuroimmunol. 184, 53-68. doi: 10.1016/j.jneuroim.2006.11.014

Xia, S. H., and Fang, D. C. (2007). Pharmacological action and mechanisms of ginkgolide B. Chin. Med. J. 120, 922-928. doi: 10.1097/00029330-200705020-00013

Xiong, N., and Wei, S. (2015). Study on protection of ginkgolide B against myocardial ischemia-reperfusion injury and its mechanism. Chin 
J. Mod. Appl. Pharm. 32, 289-294. doi: 10.13748/j.cnki.issn1007-7693.2015. 03.008

Yang, Y. H., Chen, C. Y., and Yuan, Z. P. (2018). Protective effect of ploygonati odorati rhizoma extract against mitochondrial damage and apoptosis in myocardial cells of myocardial ischemia-reperfusion injury in rats. Chin. J. Exp. Tradit. Med. Formul. 24, 136-140. doi: 10.13422/j.cnki.syfjx.20181626

Yellon, D. M., and Hausenloy, D. J. (2007). Myocardial reperfusion injury. N. Engl. J. Med. 357, 1121-1135. doi: 10.1056/NEJMra071667

Yu, S. Y., and Lu, Y. (2018). Discussion on action mechanisms of traditional Chinese medicine. Chin. J. Pharmacol. Toxicol. 32, 347-354. doi: 10.3867/j.issn.1000-3002.2018.05.001

Zhang, G. B., Chen, D. Y., Gui, C. Q., and Lu, X. H. (2005). Expermental study on protective effect of ginkgolide $\mathrm{B}$ on isolated rats' hearts with ischemia- reperfusion. Chin. J. Tradit. Med. Sci. Technol. 12, 92-94. doi: 10.3969/j.issn.1005-7072.2005.02.012

Zhang, H. F., Huang, L. B., Zhong, Y. B., Zhou, Q. H., Wang, H. L., Zheng, G. Q., et al. (2016). An overview of systematic reviews of Ginkgo biloba extracts for mild cognitive impairment and dementia. Front. Aging Neurosci. 8:276. doi: 10.3389/fnagi.2016.00276

Zhang, K. J., Zhu, J. Z., Bao, X. Y., Zheng, Q., Zheng, G. Q., and Wang, Y. (2017). Shexiang baoxin pills for coronary heart disease in animal models: preclinical evidence and promoting angiogenesis mechanism. Front. Pharmacol. 8:404. doi: 10.3389/fphar.2017.00404

Zhang, R., Xu, L., Zhang, D., Hu, B., Luo, Q., Han, D., et al. (2018). Cardioprotection of ginkgolide B on myocardialischemia/reperfusion-induced inflammatory injury via regulation of A20-NF- $\kappa$ B pathway. Front. Immunol. 9:2844. doi: 10.3389/fimmu.2018.02844

Zhao, T., Sheng, N., Su, Z. P., Wang, Q., and Ye, Y. (2014). Effects of ginkgolide $\mathrm{B}$, ginkgolide $\mathrm{C}$ and their combined therapy on the cardiacfunction of rats withischemia/reperfusion injury. Acta Acad. Med. Xuzhou. 1, 18-21.

Zhou, H., Ma, Q., Zhu, P., Ren, J., Reiter, R. J., and Chen, Y. (2018). Protective role of melatonin in cardiac ischemia-reperfusion injury: from pathogenesis to targeted therapy. J. Pineal Res. 64:e12471. doi: 10.1111/jpi. 12471

Zhuang, H. Y., Lv, J., Li, Y. L., and Shi, Y. T. (2017). Effects of ginkgolide B against apoptosis and expression of related proteins in myocardial cells of myocardial ischemia-reperfusion in rats. Jiangsu J. Trad. Chin. Med. 49, 81-84. doi: 10.3969/j.issn.1672-397X.2017.12.033

Conflict of Interest: The authors declare that the research was conducted in the absence of any commercial or financial relationships that could be construed as a potential conflict of interest.

Copyright (c) 2019 Zhu, Tong, Zhuang, Wang, Deng, Zheng and Wang. This is an open-access article distributed under the terms of the Creative Commons Attribution License (CC BY). The use, distribution or reproduction in other forums is permitted, provided the original author(s) and the copyright owner(s) are credited and that the original publication in this journal is cited, in accordance with accepted academic practice. No use, distribution or reproduction is permitted which does not comply with these terms. 